\title{
Mars Science Laboratory Mission and Science Investigation
}

\author{
John P. Grotzinger • Joy Crisp • Ashwin R. Vasavada • Robert C. Anderson • \\ Charles J. Baker · Robert Barry • David F. Blake · Pamela Conrad • \\ Kenneth S. Edgett • Bobak Ferdowski • Ralf Gellert • John B. Gilbert • \\ Matt Golombek • Javier Gómez-Elvira • Donald M. Hassler • Louise Jandura • \\ Maxim Litvak • Paul Mahaffy • Justin Maki • Michael Meyer • Michael C. Malin • \\ Igor Mitrofanov • John J. Simmonds • David Vaniman • Richard V. Welch • \\ Roger C. Wiens
}

Received: 20 December 2011 / Accepted: 30 April 2012 / Published online: 25 July 2012 (C) The Author(s) 2012. This article is published with open access at Springerlink.com

\begin{abstract}
Scheduled to land in August of 2012, the Mars Science Laboratory (MSL) Mission was initiated to explore the habitability of Mars. This includes both modern environments as well as ancient environments recorded by the stratigraphic rock record preserved at the Gale crater landing site. The Curiosity rover has a designed lifetime of at least one Mars year ( $\sim 23$ months), and drive capability of at least $20 \mathrm{~km}$. Curiosity's science payload was specifically assembled to assess habitability and includes a gas chromatographmass spectrometer and gas analyzer that will search for organic carbon in rocks, regolith
\end{abstract}

Electronic supplementary material The online version of this article

(doi:10.1007/s11214-012-9892-2) contains supplementary material, which is available to authorized users.

J.P. Grotzinger $(\bowtie)$

California Institute of Technology, Pasadena, CA, USA

e-mail: grotz@gps.caltech.edu

J. Crisp · A.R. Vasavada - R.C. Anderson - C.J. Baker · R. Barry · B. Ferdowski · J.B. Gilbert ·

M. Golombek · L. Jandura · J. Maki · J.J. Simmonds · R.V. Welch

Jet Propulsion Laboratory, California Institute of Technology, Pasadena, CA, USA

D.F. Blake

NASA Ames Research Center, Moffet Field, CA, USA

P. Conrad · P. Mahaffy

NASA Goddard Space Flight Center, Greenbelt, MD, USA

K.S. Edgett - M.C. Malin

Malin Space Science Systems, San Diego, CA, USA

R. Gellert

University of Guelph, Guelph, ON, Canada

J. Gómez-Elvira

Centro de Astrobiología (CSIC/INTA), Madrid, Spain

D.M. Hassler

Southwest Research Institute, Boulder, CO, USA 
fines, and the atmosphere (SAM instrument); an x-ray diffractometer that will determine mineralogical diversity (CheMin instrument); focusable cameras that can image landscapes and rock/regolith textures in natural color (MAHLI, MARDI, and Mastcam instruments); an alpha-particle x-ray spectrometer for in situ determination of rock and soil chemistry (APXS instrument); a laser-induced breakdown spectrometer to remotely sense the chemical composition of rocks and minerals (ChemCam instrument); an active neutron spectrometer designed to search for water in rocks/regolith (DAN instrument); a weather station to measure modern-day environmental variables (REMS instrument); and a sensor designed for continuous monitoring of background solar and cosmic radiation (RAD instrument). The various payload elements will work together to detect and study potential sampling targets with remote and in situ measurements; to acquire samples of rock, soil, and atmosphere and analyze them in onboard analytical instruments; and to observe the environment around the rover.

The 155-km diameter Gale crater was chosen as Curiosity's field site based on several attributes: an interior mountain of ancient flat-lying strata extending almost $5 \mathrm{~km}$ above the elevation of the landing site; the lower few hundred meters of the mountain show a progression with relative age from clay-bearing to sulfate-bearing strata, separated by an unconformity from overlying likely anhydrous strata; the landing ellipse is characterized by a mixture of alluvial fan and high thermal inertia/high albedo stratified deposits; and a number of stratigraphically/geomorphically distinct fluvial features. Samples of the crater wall and rim rock, and more recent to currently active surface materials also may be studied. Gale has a well-defined regional context and strong evidence for a progression through multiple potentially habitable environments. These environments are represented by a stratigraphic record of extraordinary extent, and insure preservation of a rich record of the environmental history of early Mars. The interior mountain of Gale Crater has been informally designated at Mount Sharp, in honor of the pioneering planetary scientist Robert Sharp.

The major subsystems of the MSL Project consist of a single rover (with science payload), a Multi-Mission Radioisotope Thermoelectric Generator, an Earth-Mars cruise stage, an entry, descent, and landing system, a launch vehicle, and the mission operations and ground data systems. The primary communication path for downlink is relay through the Mars Reconnaissance Orbiter. The primary path for uplink to the rover is Direct-from-Earth. The secondary paths for downlink are Direct-to-Earth and relay through the Mars Odyssey orbiter.

Curiosity is a scaled version of the 6-wheel drive, 4-wheel steering, rocker bogie system from the Mars Exploration Rovers (MER) Spirit and Opportunity and the Mars Pathfinder Sojourner. Like Spirit and Opportunity, Curiosity offers three primary modes of navigation: blind-drive, visual odometry, and visual odometry with hazard avoidance. Creation of terrain maps based on HiRISE (High Resolution Imaging Science Experiment) and other remote

M. Litvak · I. Mitrofanov

Space Research Institute (IKI), Moscow, Russia

M. Meyer

NASA Headquarters, Washington, DC, USA

D. Vaniman

Planetary Science Institute, Houston, TX, USA

R.C. Wiens

Los Alamos National Laboratory, Los Alamos, NM, USA 
sensing data were used to conduct simulated driving with Curiosity in these various modes, and allowed selection of the Gale crater landing site which requires climbing the base of a mountain to achieve its primary science goals.

The Sample Acquisition, Processing, and Handling ( $\mathrm{SA} / \mathrm{SPaH})$ subsystem is responsible for the acquisition of rock and soil samples from the Martian surface and the processing of these samples into fine particles that are then distributed to the analytical science instruments. The SA/SPaH subsystem is also responsible for the placement of the two contact instruments (APXS, MAHLI) on rock and soil targets. SA/SPaH consists of a robotic arm and turret-mounted devices on the end of the arm, which include a drill, brush, soil scoop, sample processing device, and the mechanical and electrical interfaces to the two contact science instruments. SA/SPaH also includes drill bit boxes, the organic check material, and an observation tray, which are all mounted on the front of the rover, and inlet cover mechanisms that are placed over the SAM and CheMin solid sample inlet tubes on the rover top deck.

Keywords Mars $\cdot$ Curiosity $\cdot$ Rover $\cdot$ Gale $\cdot$ Mount Sharp

\section{Mission Perspective: Beyond Water on Mars}

\subsection{Mars Exploration}

The exploration of Mars has never been more active than at this moment. The Opportunity rover and three orbiters-Mars Odyssey, Mars Express, and Mars Reconnaissance Orbiter (MRO) routinely analyze the current and past states of the planet's environment. The Spirit rover forged a trail of discoveries leading into the heart of hydrothermal processes in the early history of Mars. These recent missions build systematically upon the earlier Mars Global Surveyor orbiter mission and the Pathfinder proof-of-concept lander and rover mission. Discoveries from Odyssey spawned the follow-up lander, Phoenix. It is a remarkable achievement that each of these recent missions has functioned so capably, performing well past their nominal operational periods, all with spectacular results. But what really impresses - with all the hardware in motion around Mars these days - is the high degree of both tactical and strategic coordination among these missions, which has propelled us ever closer to fathoming the broad range of environmental processes that transformed the surface of Mars, beginning over 4 billion years ago (Fig. 1).

The unexpected dividends of these extended, overlapping, and increasingly coordinated missions are rich. For the earlier part of Mars history this includes recognition of very ancient basaltic crust altered by aqueous processes (Ming et al. 2008) to produce diverse assemblages of hydrated phyllosilicate minerals (Mustard et al. 2008; Poulet et al. 2005); the discovery of vast sequences of thick, well-bedded sedimentary rocks of largely unknown origin and composition but in some places clearly containing hydrated sulfates and/or phyllosilicates, hematite, and opaline silica (Malin and Edgett 2000; Edgett and Malin 2002; Glotch et al. 2006; Grotzinger and Milliken 2012); the recognition of many local topographic depressions - craters and structural troughs_-filled with alluvial fans and deltas that contain hydrated phyllosilicates (Malin and Edgett 2003; Moore and Howard 2005; Ehlmann et al. 2008); and the revelation that Mars has a rich and varied history characterized by a rock cycle involving accumulation of sediments, their burial, alteration and transformation, followed by exhumation and their return to the surface (McLennan and Grotzinger 2008; Grotzinger and Milliken 2012). The summary conclusion from all these discoveries is that 


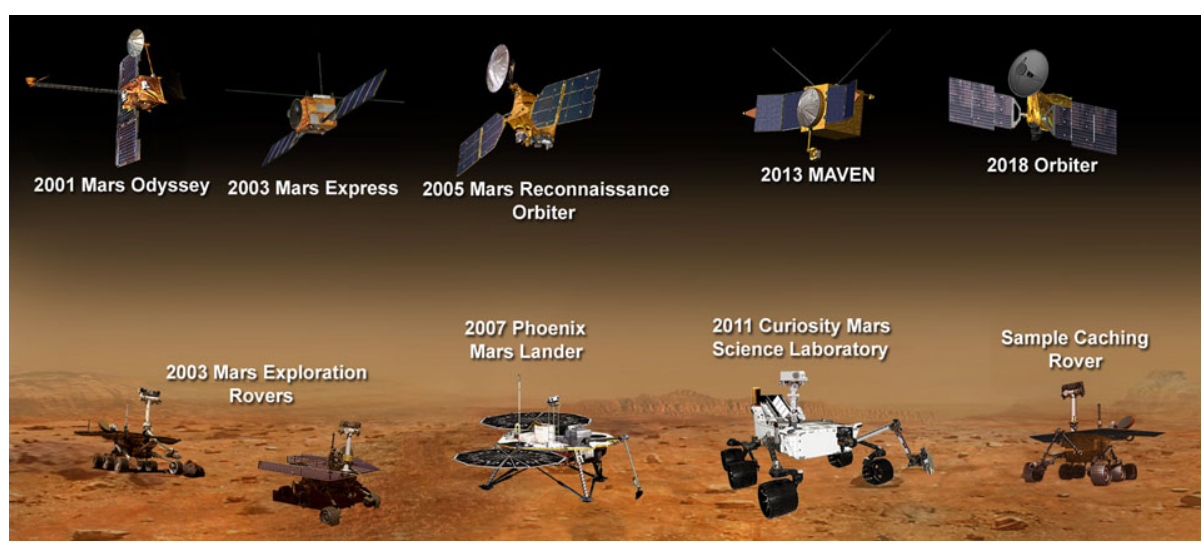

Fig. 1 Currently operating and future potential missions to Mars. This program architecture provides a high degree of synergy and scientific leverage between missions. MSL will benefit from both previous and concurrent missions

the surface of Mars has been transformed by interactions with water throughout its history. This is exciting for science, but also reassuring to the Mars Program officials who adopted the "follow-the-water" strategy. This strategy has worked well and the planetary science community is richer for it. At this point a natural question to ask is, "What's next?"

\subsection{Habitability and Preservation}

Mars Science Laboratory (MSL) was designed to address this question and will undertake the search for past and present habitable environments at Gale crater. Loosely defined, a habitable environment is one that has liquid water, a source of carbon (to enable organism metabolism), and a source of energy (to fuel organism metabolism) —in other words, the essential ingredients for life as we know it on Earth. To search for these essential ingredients on our nearest neighbor planet constitutes a logical next step in the ultimate goal of searching for life elsewhere in our solar system and beyond.

To be clear, MSL is not a life detection mission and has no capability to detect extant vital processes that would betray present-day microbial metabolism. Nor does it have the ability to image microorganisms or their fossil equivalents. MSL does have, however, the capability to detect complex organic molecules in rocks and soils. If present, these might be of biological origin, but could also reflect the influx of carbonaceous meteorites. More indirectly, MSL will have the analytical capability to probe other less unique biosignatures, specifically, the isotopic composition of inorganic and organic carbon in rocks and soils, particular elemental and mineralogical concentrations and abundances, and the attributes of unusual rock textures. The main challenge in establishment of a biosignature is finding patterns, either chemical or textural, that are not easily explained by physical processes (Knoll 2003). MSL will also be able to evaluate the concentration and isotopic composition of potentially biogenic atmospheric gases such as methane, which may be present in the modern atmosphere (Atreya et al. 2007; Mumma et al. 2009). But compared to the current and past missions that have all been targeted to find evidence for past or present water, the task of searching for habitable environments is significantly more challenging. Mostly, this is because it is unknown to what degree organic carbon would be preserved on the Martian surface-even if it were produced in abundance. Organic carbon is a reduced compound 


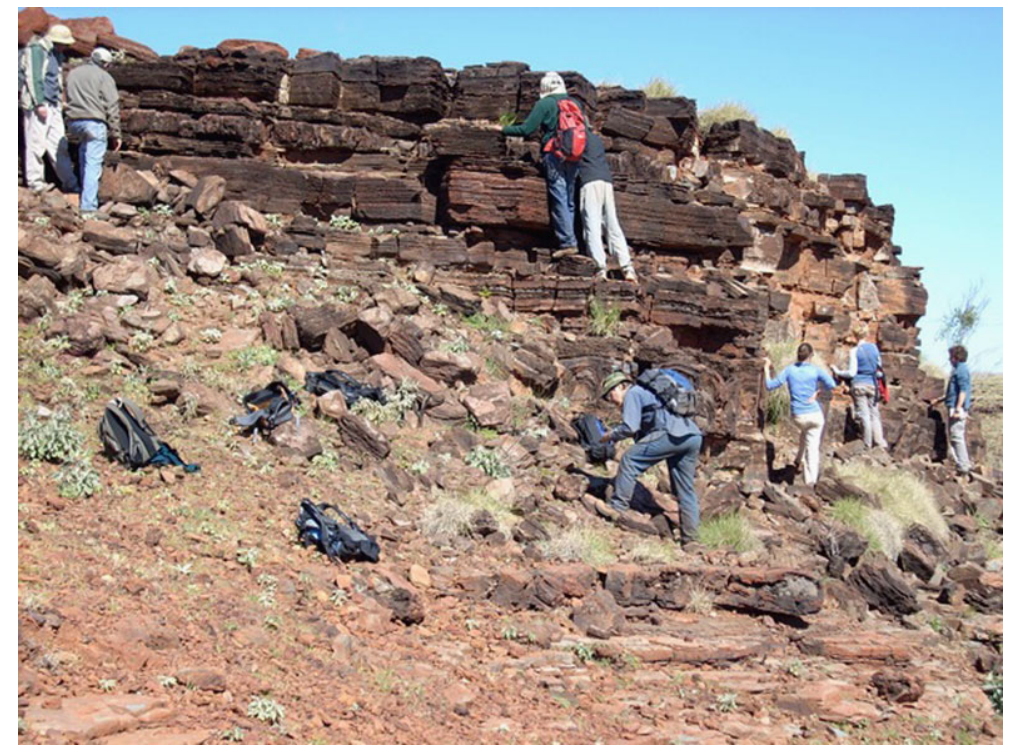

Fig. 2 Sedimentary rocks billions of years old on Earth form the principle repository of geochemical and textural data that allow determination of habitability on the young Earth. To discover preserved organic compounds field studies must carefully focus on those successions of layered rocks which show evidence of accumulation in subaqueous environments, and where very early precipitation of minerals such silica, carbonate, sulfate, clay, or phosphate has occurred. Outcrop of 2.7 billion year old Tumbiana Formation, Western Australia, 2007

and is expected to have a short lifetime given that the current Martian surface environment is known to contain a variety of oxidants (Hunten 1979; Sumner 2004; Navarro-Gonzalez et al. 2003). Furthermore, many diagenetic environments, where most biosignatures enter the rock record by becoming coated in stable minerals, involve circulation of oxidizing fluids that could decompose organic matter. Thus MSL will be faced with a major challenge: both modern weathering processes (including radiation damage) and ancient diagenetic processes could conspire to inhibit the preservation of organic matter.

If we employ Earth's early geologic record as a guide to prediction of biosignature preservation in the ancient Martian rocks to be sampled by MSL then we should prepare to be patient. Assuming microbes were once present on Mars, we will also need to focus on very specific and systematic exploration strategies. Scientists working on the terrestrial record of early life long ago recognized to study those rocks whose preservation character maximizes the chances of success. Paleontological exploration is critically sensitive to the diagenetic processes that control preservation and, paradoxically, the very characteristics (water, gradients in heat, chemicals, and light, and also oxidant supply) that make so many environments habitable also cause them to be destructive to biosignature preservation. Nevertheless, though most habitable environments destroy organic materials, there are rare circumstances that facilitate spectacular preservation; these often involve geochemical conditions that favor very early mineralization. Authigenic silica, phosphate, clay, sulfate, and less commonly, carbonate precipitation are all known to promote biosignature preservation when all other factors, such as environmental redox conditions, are equal (Fig. 2).

Therefore, if MSL is to succeed in detecting organic compounds this will require a habitable environment that also favors preservation of organic compounds. This search can be 
optimized by pursuing an exploration strategy that focuses on the search for windows of preservation. We should be guided but not limited by our terrestrial experience, lest we forget that Mars may indeed have its own unique paleoenvironmental conditions favorable to the preservation of organic compounds and other potential biosignatures. It will be MSL's task to identify the characteristics of these environments and where they can be found.

\subsection{Environmental Records}

An essential point that Earth also teaches us is that in the search for signs of early life a null result is a not always a disappointment. Whatever may be lost in terms of insight into possible paleobiologic markers may be gained by an equally rich reward into the processes and history of early environmental evolution. Studies of Earth's Precambrian sedimentary record have revealed secular changes in the oxidation state, acid-base chemistry, and precipitation sequence of minerals in the oceans and atmosphere (Des Marais 2001; Knoll 2003; Hazen et al. 2008). Knowledge of an equally informative environmental history may also be uncovered on Mars. The evolutionary path of surface environments on an Earth-like planet that lacked a biosphere would make a highly desirable comparison to Earth in order to understand better the unique aspects of our own planet's history. These records of environmental history are also embedded within the same kinds of rocks and minerals that may also preserve the calling cards of biology. Therefore, an MSL mission that focuses on understanding mechanisms of potential biosignature preservation will also insure that we capture the record of early Martian environmental processes and history.

This approach holds both the hope and promise of Mars Science Laboratory. The hope is that we may find some signal of a biologic process. The promise is that MSL will deliver fresh insight into the comparative environmental evolution of the early stages of Mars and Earth. That alone is a valuable prize. MSL was specifically designed for this purpose and the MSL team has a lot going for it: veterans of years of previous rover operations permeate the engineering and science teams; strategic decision making has already benefited from stunning high-resolution image datasets obtained by the HiRISE camera on MRO, as applied to both drive-related terrain assessment at Gale crater and refinement of scientific objectives; and the rover itself will be the most capable robot ever sent to the surface of another planet. The MSL payload was specifically assembled for the purpose of environmental (and paleoenvironmental) assessment.

The Gale crater landing site gives MSL a good head start on the search for past habitable environments that could preserve paleoenvironmental indicators. While each of the four final landing sites had its own particular strengths, they all shared in common two very important attributes: definitive evidence for the former presence of water as seen by either mineralogic or morphologic features (or both), and the presence of prominent stratigraphic sequences, hundreds to thousands of meters thick in some cases, suggestive of sedimentary rocks (Fig. 3). Historical accounts of planetary evolution are largely recorded in its rock record, and processes that operate at a planetary surface have the potential to create a record of sedimentary rocks. This is important because our experience on Earth shows that sediments and sedimentary rocks can preserve high-resolution proxies of present and past climatic, tectonic, and biological processes as well as providing the dominant archive of major events in Earth's evolution. Sedimentary rocks precipitated from water are particularly important because they embed signals of elemental and isotopic variability that relate to geochemical and biogeochemical processes, expressed at local to global scales. Although other rock types such as hydrothermal deposits within volcanic terrains also hold potential to be both habitable and favorable for preservation of biosignatures, terrestrial experience 

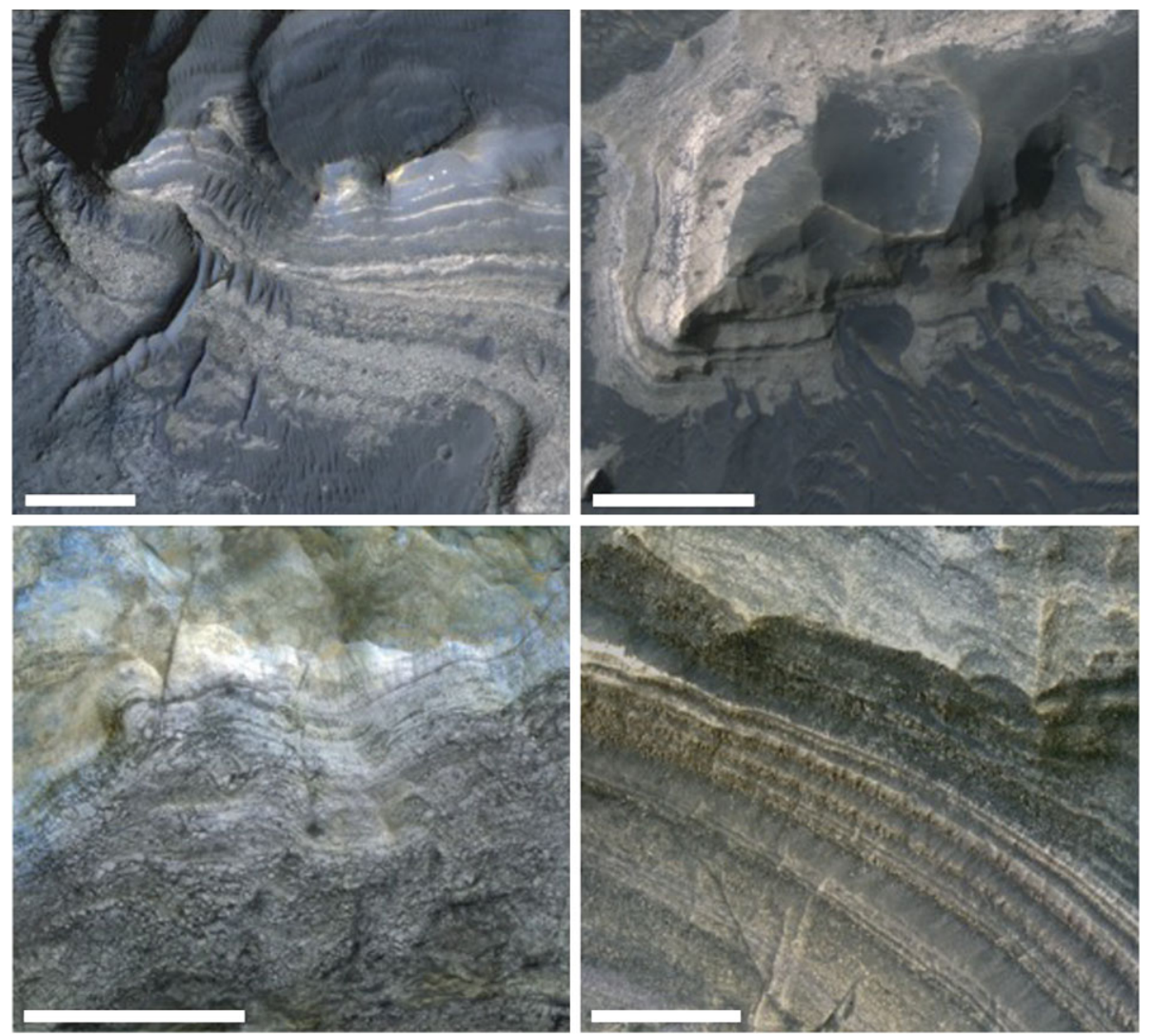

Fig. 3 HiRISE images of the four finalist MSL landing sites include: (a) Eberswalde crater. Strata shown are near the base of a delta deposit; (b) Holden crater. Strata shown also represent inferred flood deposits; (c) Mawrth Vallis. Strata shown are near the top of several hundred meter-thick deposit and preserve a transition from iron-magnesium phyllosilicates (darker tone) to aluminum-rich phyllosilicates (lighter tone); (d) Gale crater. Strata shown are near the base of a 5-kilometer thick succession. Gale strata also contain interbedded sulfate deposits. All scale bars are $100 \mathrm{~m}$. Top left (Eberswalde crater), PSP_001336_1560 (FYI, this image is rotated so north is down); Top right (Holden crater), PSP_001666_1530; Bottom left (Mawrth Vallis), PSP_005964_2045; Bottom right (Gale crater), PSP_006855_1750. Images courtesy of NASA/JPL/U. Arizona

shows that sedimentary rocks are the favored medium for preservation of both biosignatures and global environmental records. Considering all these factors the MSL Science Team and MSL Project Science Group (PSG) preferred Gale crater as the site most suitable for the MSL science investigation. These considerations were based on years of study of data sets unprecedented before MRO's arrival in 2006.

Though the precise nature of the geochemical interactions between the Martian crust, hydrosphere, and atmosphere will occupy planetary scientists for years to come, MSL will provide the first step toward a more detailed understanding of the key processes and environmental transitions, and their relevance for evaluating microbial habitability and the preservation potential of possible biosignatures and environmental proxies. Measurement of the first long-term, high-resolution stratigraphic records of early environmental processes at Gale crater would fulfill the promise of MSL. 




Fig. 4 Curiosity (flight) rover in Spacecraft Assembly Facility at the Jet Propulsion Laboratory with robotic arm deployed

\section{Mission Overview}

\subsection{MSL Mission Summary}

The Mars Science Laboratory Mission will explore and quantitatively assess the habitability and environmental history of the Gale crater field site (landing ellipse, adjacent lower portion of Mount Sharp). As described in the 2004 Announcement of Opportunity solicitation, the mission has the primary objective of placing a mobile science laboratory on the surface of Mars to assess the biological potential of the landing site, characterize the geology of the landing region, investigate planetary processes that influence habitability, and characterize the broad spectrum of surface radiation. The MSL Project aims to achieve this objective in a manner that will offer the excitement and wonder of space exploration to the public. Figure 4 shows the Curiosity rover in Assembly, Test, and Launch Operations (ATLO) at JPL, with its instrument arm deployed. Figure 5 shows Curiosity during environmental testing.

The mission was launched on November 26, 2011, on an Atlas V 541 from Cape Canaveral Air Force Station in Florida. The baseline plan was for the launch to occur between 25 November 2011 and 18 December 2011, with an arrival date at Mars of 6 August 2012 (UTC). The primary science mission is one Mars year (669 Martian diurnal cycles, called "sols", or 687 Earth days). Further details about the launch, cruise, approach, entry, descent, and landing are described in Abilleira (2010) and Steltzner et al. (2010).

The major subsystems of the MSL Project consist of a single rover (with science payload), a Multi-Mission Radioisotope Thermoelectric Generator (MMRTG; see Fig. 5), an Earth-Mars cruise stage, and entry, descent, and landing system, a launch vehicle, and the mission operations and ground data systems. The primary communication path for downlink is relay through the Mars Reconnaissance Orbiter (MRO). The secondary paths for downlink are Direct-to-Earth and relay through the Mars Odyssey orbiter. The primary path for uplink to the rover is Direct-from-Earth (DFE). The secondary path for uplink is relay through MRO. 


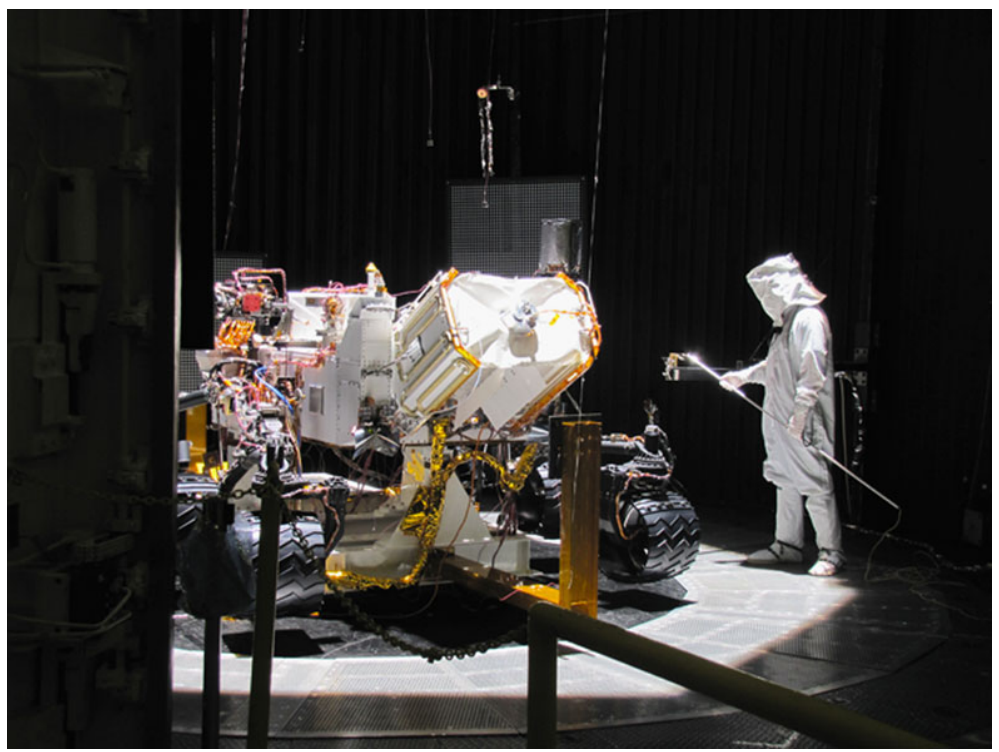

Fig. 5 Curiosity in System Thermal Testing facility at JPL. Rearward perspective shows location of MultiMission Radioisotope Thermoelectric Generator

The Mars Science Laboratory will begin surface operations soon after landing in early August 2012 and continue for at least one Mars year (approximately two Earth years). The overall scientific goal of the mission is to explore and quantitatively assess a local region on Mars' surface as a potential habitat for life, past or present. The MSL rover carries ten scientific instruments and a sample acquisition, processing, and distribution system. The various payload elements will work together to detect and study potential sampling targets with remote and in situ measurements; to acquire samples of rock, soil, and atmosphere and analyze them in onboard analytical instruments; and to observe the environment around the rover.

These observations and measurements will individually be of great scientific interest and importance, but the overall scientific goal of assessing present and past habitability of environments at the visited sites will only come from their comprehensive integration, and this is consequently a key feature of the proposed mission. Each of the ten instruments contributes to multiple science objectives, and most of the science objectives involve contributions from several instruments. Because of the cross-instrument nature of the science return, much of the tactical operations and science assessment will be coordinated through science theme groups comprising the entire MSL science team, as discussed in a later section. Strategic science operations, data analysis, and dissemination of results will be integrated by and coordinated through the MSL Project Science Group (PSG).

\subsection{MSL Science Team}

The MSL Science Team currently consists of the instrument investigation PIs, Co-Is, Collaborators, Project Scientist, Program Scientist, Deputy Project Scientists, and Investigation Scientists. Participating Scientists were selected in November of 2011. The MSL Science 
Table 1 Mars Science Laboratory Project Science Group

\begin{tabular}{lll}
\hline Name & Role & Affiliation \\
\hline John Grotzinger & Project Scientist & California Institute of Technology \\
Michael Meyer & Program Scientist & NASA Headquarters \\
David Blake & PI, CheMin & Ames Research Center \\
Kenneth Edgett & PI, MAHLI & Malin Space Science Systems \\
Ralf Gellert & PI, APXS & University of Guelph, Canada \\
Javier Gómez-Elvira & PI, REMS & Centro de Astrobiología/INTA, Spain \\
Donald Hassler & PI, RAD & Southwest Research Institute \\
Paul Mahaffy & PI, SAM & Goddard Space Flight Center \\
Michael Malin & PI, MARDI and Mastcam & Malin Space Science Systems \\
Igor Mitrofanov & PI, DAN & Space Research Institute, Russia \\
Roger Wiens & PI, ChemCam & Los Alamos National Laboratory \\
\hline
\end{tabular}

Team Rules of the Road document (see Supplementary Information) provides the principles, ground rules, and operations policies that will underpin the Project's approach to managing the integrated scientific investigations, including data access, data sharing, data release, publication authorship privileges, and integrated instrument operations. All MSL Science Team members are expected to adhere to the Rules of the Road and any future updates approved by the PSG.

The PSG is co-chaired by the MSL Project Scientist and the Mars Program Scientist and comprises the instrument PIs as members. The primary function of the PSG is to advise the Project on optimization of mission science return and on resolution of issues involving science activities. During landed operations, the PSG will have an important role providing strategic guidance to the Science Operations Working Group (that subset of MSL science team members on shift making tactical decisions on any given sol of the mission). The list of MSL PSG members is shown in Table 1.

During development, the MSL Science Office management consisted of the Project Scientist (John Grotzinger) and Deputy Project Scientists (Joy Crisp, Ashwin Vasavada). Significant responsibilities were distributed, without any one person dominating the effort. These responsibilities included supervision of: mission science objectives, mission science performance, PSG working groups, Program interactions, instrument development and calibration, data archiving, export compliance, contamination control, sample library, EDL characterization, surface environments, mission scenarios, landing site selection and certification, science operations and training, science team planning (logistics, staffing), and education and public outreach. This contrasts with PI-led missions in which a single PI may tend to lead many of these key tasks. In part the size of MSL dictates this distribution of effort, however, it was also by choice in design that the Science Office architecture was set up this way, as a different management model to explore. To a limited extent it represents a model implemented by the Viking mission, where an integrated science payload was used on a daily basis. The mobility system of MSL, however, strongly differentiates it from the Viking landers and the science return of each payload element depends on the course the rover will take over its exploration campaign.

Also part of the Science Office, a number of Investigation Scientists served as liaisons between the Project and the external instrument teams. A separate Payload Office, led by J. Jeff Simmonds, managed the development and delivery of the instruments. A team of Instrument Engineers served as liaisons to the external teams on technical and interface 
issues, complementing the role of the Investigation Scientists. Finally, a Mission System office managed by Michael Watkins led the planning for surface operations. After launch, the organization condensed down to a single Science Office, managed by Ashwin Vasavada, retaining the above staff and enveloping the Payload Manager. The role of Science Operations Team Chief, staffed by Nicole Spanovich, is the executive lead for science operations.

\subsection{Gale Crater Field Site}

The MSL "field site" (landing ellipse plus adjacent areas to be explored) at Gale crater is expected to profoundly influence the nature and quality of the scientific return from the mission, as well as the pace and strategy for surface operations. Gale contains evidence suggestive of potentially diverse habitable environments. In addition, it exceeded all engineering and safety constraints including operational performance.

The geology, mineralogy, context, and description of potential exploration targets of the Gale crater field area are presented in Malin and Edgett (2000), Anderson and Bell (2010), Milliken et al. (2010), and Thomson et al. (2011). A broader context for understanding Mount Sharp is developed in Grotzinger and Milliken (2012). Gale is a heavily degraded crater, about $150 \mathrm{~km}$ in diameter that straddles the dichotomy boundary. The dichotomy boundary - a sharp global-scale contrast in topography-in this region of Mars is crossed by numerous incised valley networks suggestive of surface aqueous flows that discharged across the proximal northern lowlands. The $\sim 5 \mathrm{~km}$ in thickness of strata that comprise Mount Sharp occupies the center of the crater, and strata at the base of Mount Sharp contain hydrated minerals indicative of aqueous alteration processes. Thus, the regional context is one that provides a high degree of confidence that Curiosity will discover features of importance to understanding past habitability.

Descriptions of the process of selecting the landing site and certifying the safety of the site terrain and atmospheric conditions can be found in Golombek et al. (2012, this issue) and Vasavada et al. (2012, this issue). The selection of the MSL landing site was informed through a series of community-led open workshops that occurred in parallel with the design and development of the spacecraft. A series of meetings involving the MSL Science Team, the MSL PSG, and NASA HQ resulted in the eventual selection of the Gale crater landing site. The selecting official was the NASA Associate Administrator for the Science Mission Directorate.

The full Science Team was involved in independent analysis and consideration of the various landing sites. These activities were created due to the very sophisticated nature of the mission goals, involving multiple objectives depending on coordinated observations between many instruments. The PSG co-chairs (Project Scientist and Program Scientist), in consultation with the PIs determined that analysis of the landing sites would be aided by the involvement of the MSL Science Team, who would be intimately familiar with the instruments and objectives of the mission. These discussions became instrumental in defining the mission-relevant landing science criteria used by the broader Mars community in framing discussion of the candidate landing sites (Table 2).

It was decided to charter three PSG Working Groups, each operating under the auspices of the PSG co-chairs. The first of these was chartered to specifically look at the preservation potential of organic compounds and other biosignatures on Mars, as a function of different habitable environments thought to be present at the different landing sites as presented by the community. The second Working Group was chartered to lead comprehensive discussions and analysis of the final four landing sites. The third Working Group was charged with evaluating the traversability of the rover through sloping outcrops of the lower part of Mount Sharp. Where needed the Working Groups were inclusive of experts external to the Project and Science Team, so as to maximally enhance the return and objectivity of these efforts. 
Table 2 Four major mission relevant landing site science criteria

Criteria Description

Diversity A site with a variety of possible science objectives will ensure a greater chance for scientific success. Examples: multiple and differentiated science targets, multiple types of evidence (e.g., morphologic and geologic), variety in mineralogy or styles of stratigraphic expression.

Context A site that can be placed in a larger, more regional context will ensure a greater depth of scientific understanding. The regional context provides constraints on past processes that led to the environments being examined locally. Locally derived results can, in turn, be extrapolated regionally or globally.

Habitability Sites with orbiter-derived evidence for habitable environments can be assessed to make specific predictions that will guide the exploration strategy for MSL. Particular high-priority geologic targets can be identified that can be accessed, interrogated, and interpreted by MSL.

Preservation Sites with a higher potential for preserving evidence for past habitable environments will ensure a greater chance of scientific success. Using terrestrial analogs, sites can be assessed for the particular physical and chemical conditions that retain mineralogic, chemical, or morphologic evidence.

Fig. 6 Key science targets at Gale crater shown on CTX mosaic. See text for discussion. Center lat/lon of ellipse is $4.49 \mathrm{~S}$, 137.42 E. Ellipse is $20 \times 25 \mathrm{~km}$ for scale. Images used in mosaic: B07_012195_ 1750_NS_05S222W, B01_ 009927_1752_XN_04S222W, and B21_017786_1746_ SN_05S222W. CTX data courtesy of NASA/JPL/MSSS

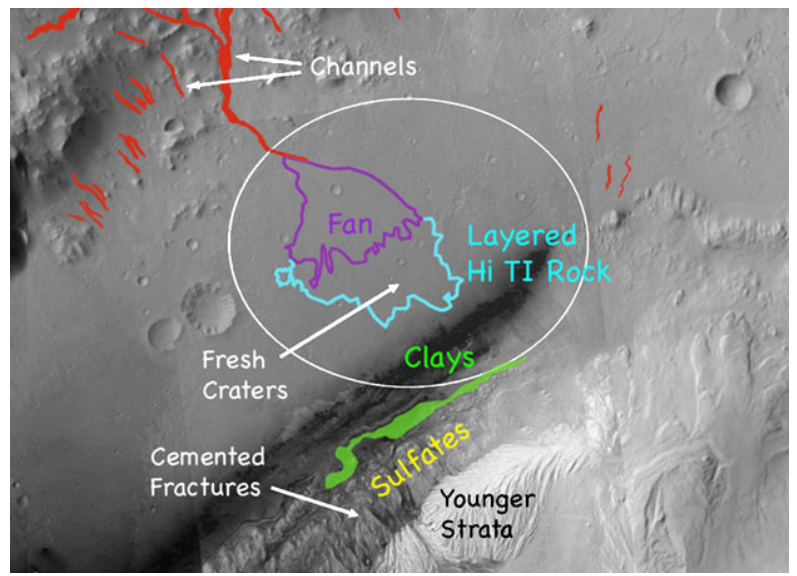

\subsubsection{Examples of Gale Science Targets}

The landing ellipse and adjacent mountain of strata comprise the region to be explored by the Curiosity rover (Fig. 6). In order of increasing distance from the center of the landing ellipse, several targets of particular interest include: (1) an alluvial fan near the center of the ellipse; (2) a layered rock body with high thermal inertia and high albedo similar to cemented sedimentary materials elsewhere on Mars (Fergason et al. 2012, this issue); (3) fresh craters that penetrate this high thermal inertia unit; (4) strata within the lowermost part of Mount Sharp that contain enrichments in hydrated clay minerals; (5) strata throughout the lower part of Mount Sharp that contain enrichments in hydrated sulfate minerals; and (6) strata near the top of the lower part of Mount Sharp that contain large fracture networks filled with light-toned minerals.

These features create a diverse set of science targets that meet the challenge of the mission objectives, described earlier in this paper. The alluvial fan, high thermal inertia unit, 


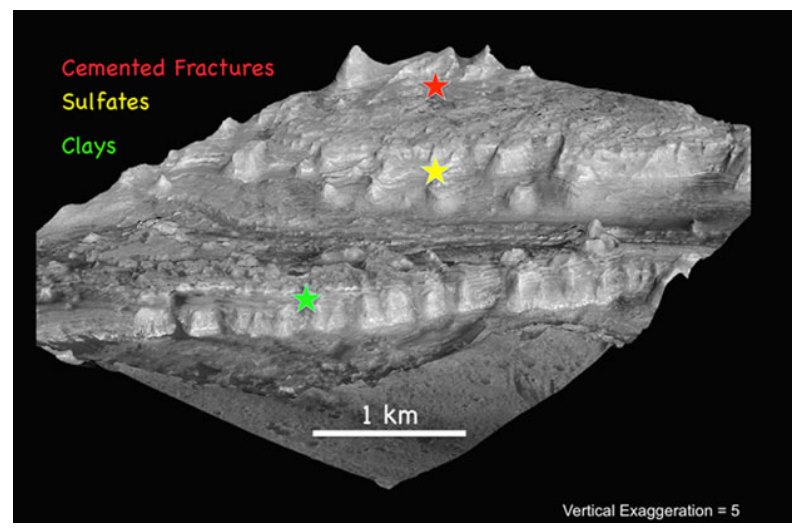

Fig. 7 Digital Elevation Model of strata exposed at the base of Mount Sharp, located in the center of the crater. Strata are composed of sulfate minerals, mixed with concentrations of clays that form distinct layers. Toward the top of this succession bedding plane exposures reveal plan-view cross-sections of fracture networks filled by minerals (see Fig. 8). DEM constructed by USGS, based on HiRISE images PSP_001488_1750 and PSP_001752_1750. HiRISE data courtesy of NASA/JPL/U. Arizona

Fig. 8 HiRISE image showing bedding plane view of filled fractures near top of stratigraphic interval defined by enrichment in sulfate minerals. Fractures are filled with later generations of minerals, suggesting precipitation in subsurface (groundwater) setting. HiRISE image PSP_001488_1750. HiRISE data courtesy of NASA/JPL/U. Arizona



clay-rich strata, sulfate-rich strata, and fracture fill networks present a variety of potentially habitable environments to explore regarding habitability and possibly the preservation of organic carbon. Curiosity will be able to test a range of different surface environments, in addition to the subsurface environment presented by the fracture fills. Furthermore, the fresh craters offer the chance to conduct a set of experiments related to the effects of radiation exposure on preservation processes; the ejecta blocks will likely have seen a lesser degree of radiation exposure as compared to adjacent surface rocks.

Having left the landing ellipse, Curiosity will be commanded to drive up through the outcropping ridges, slopes and buttes that define the lower part of Mount Sharp. These outcrops expose the lower strata of Mount Sharp, composed of interbedded clays and sulfates (Fig. 7). Near the top of the interval of strata that contains well-defined sulfates are a set of bedding planes that expose plan-view cross-sections through cement-filled fractures of very large (decameter) scale (Fig. 8). Comparison to similar terrestrial features suggests that groundwater circulated through these fractures, providing dissolved minerals that precipitated along the margins of the fractures eventually occluding much of their initial porosity. 


\subsection{Biosignature/Carbon Compound Preservation Working Group}

In order to help engage the full MSL science team with the challenge of searching for organics with Curiosity, a PSG Working Group was chartered to help establish a process by which different landing sites could be evaluated for their potential to preserve biosignatures including carbon compounds, in addition to habitable environments. In a broad sense the case for habitable environments at many of the proposed MSL landing sites is reasonably strong based on existing orbiter and landed mission data that have demonstrated the role of past water in mineral precipitation and landscape/stratigraphic evolution. What is less clear is how favorable these possibly habitable environments are for preservation of recognizable biosignatures, or abiotic organic compounds - chemical, mineralogic, and morphologic. It is important to note here that the discovery of actual biosignatures or abiotic organic compounds is not a requirement for mission science success, or even a strong expectation. Indeed, the recognition of how difficult it is to discover evidence of compelling biosignatures/carbon compounds on the early Earth tempers our expectations for Mars. But within this sobering context comes an equally compelling reminder that in the investigation of early biosignatures on Earth, the loss of insight regarding biology is almost always compensated by the gain of insight into the history and range of processes of early surface environments. So by pursuing a similar strategy with MSL we couple a clear strategic vision for landed surface operations with the promise of important discoveries regarding the early evolution of Mars.

The charge of the Biosignature/Carbon Compound Working Group was to assess the potential diversity of biosignature/organic compound preservation windows that may be recorded in the ancient terrains that MSL might be exploring (Table 3). This was established as a two-step process that first considered the range of possibilities in a general sense, followed by a more focused evaluation which considered specific opportunities identified at the candidate landing sites, as well as instrument capabilities and detection limits.

The Working Group included both MSL Team and non-team members (Table 4). Their findings were published in the journal Astrobiology (Summons et al. 2011). These results were very helpful in educating the MSL Science Team members in terms of what constitute the most likely targets for potential preservation of organic compounds, had they been present on the surface of Mars. Of the many categories of rock types listed by Summons et al. (2011) sedimentary rocks formed in evaporitic lacustrine settings were considered to have the highest preservation potential.

\subsection{Landing Site Working Group}

Experience from previous rover missions (MER, Pathfinder) suggested that at least some favored MSL landing sites might be rejected because of engineering constraints. However, as the MSL Project continued to study the final four landing sites it became increasingly clear that all landing sites would be deemed satisfactory for landing given engineering requirements. Given this possibility a decision was made to start a process to supplement the community workshops with a more continuous, ongoing analysis of all the science data. If science alone was to be the final discriminant in the eventual decision then it was judged to be of utmost importance for the Science Team to become as familiar as possible with all data and published interpretations. A PSG Working Group was tasked with this process: to examine all sites as thoroughly as possible from the perspective of observations, suggested hypotheses, and goodness-of-fit to the mission objectives.

A steering committee was formed to oversee activities of the Landing Site Working Group (LSWG, see Table 5). LSWG membership included all team members, in addition to 
Table 3 Summary of what biosignatures could be observed with the payload elements (PE) of MSL. Note that environments can be reconstructed from physical and chemical features of ancient sediments that are not considered to be biomarkers. Modified from Summons et al. (2011)

1

Organism Morphologies (cells, body fossils, casts)

\section{PE: MAHLI}

Minimum size would have to be greater than $100 \mu \mathrm{m}$ and rock preparation techniques are not available to expose organisms within rock. Possible Martian organisms are expected to be microbial, so the probability of detection is low

Potential as a biosignature: exceptionally high

Potential as an environmental indicator: low

Biofabrics (including stromatolites)

PE: MAHLI, MastCam

Accreted structures analogous to those on Earth are detectable; Cross-sections of strata and bedding plane surfaces are required; potential biosignatures could be detected driving across the strata and their associated exposed bedding planes at Gale crater

Potential as a biosignature: moderate

Potential as an environmental indicator: low

Diagnostic organic molecules; Organic carbon

PE: SAM, \pm ChemCam only if very abundant. Detection potential high including atmospheric gases

Potential as a biosignature: exceptionally high

Potential as an environmental indicator: high

PE: SAM

Contextual knowledge is essential; results can be ambiguous and complex to interpret

Potential as a biosignature: moderate

Potential as an environmental indicator: low

Biomineralization \& bioalteration

PE: CheMin, \pm MAHLI, \pm SAM, \pm APXS

Detection of specific minerals is good

Morphological pattern may be useful but needs very fine spatial resolution

Potential as a biosignature: low

Potential as an environmental indicator: low

Spatial patterns in chemistry

PE: SAM, CheMin, \pm ChemCam if very abundant

C, N, S elemental distributions; Detection potential on $\mathrm{cm}$ scale to facies scale

Potential as a biosignature: low on its own

Potential as an environmental indicator: low

site advocates from the external community. Over the course of 9 months leading up to the September 2010 community workshop, on the order of 50 telecons were held that added up to over 100 hours of discussion by $20-50$ participtants. In addition, a series of "tiger teams" were created to study specific aspects of the landing sites in more detail (Table 6). These tiger teams involved smaller numbers of discussants, again including members external to the MSL Science Team. Tiger teams were self-organized and their leaders reported out to the entire Science Team at all-hands science team meetings.

While there were many benefits of having established the LSWG perhaps the most significant was that MSL Science Team members felt that with the benefit of this extra discussion they were ready to converge, very rapidly, on recommendations to advance regarding the 
Table 4 Biosignature/Carbon Compound Working Group

${ }^{\mathrm{a} C o m m i t t e e ~ c h a i r ~}$

Table 5 Landing Site Working Group Steering Committee

${ }^{\mathrm{a} C o m m i t t e e ~ c o-c h a i r s ~}$

Table 6 Landing Site Assessment Tiger Teams

\begin{tabular}{ll}
\hline Name & Affiliation \\
\hline Roger Summons $^{\mathrm{a}}$ & Massachusetts Institute of Technology \\
Jan Amend & Washington University, St. Louis \\
Roger Buick & University of Washington \\
George Cody & Carnegie Institution \\
David DesMarais & Ames Research Center \\
Gilles Dromart & École Normale Supérieure de Lyon \\
Jennifer Eigenbrode & Goddard Space Flight Center \\
Andrew Knoll & Harvard University \\
David Bish & University of Indiana \\
Dawn Sumner & University of California, Davis \\
\hline
\end{tabular}

\begin{tabular}{ll}
\hline Name & Leader \\
\hline Mineralogy & David Vaniman \\
Impactites & Horton Newsom \\
Radiation and Organics & Pamela Conrad \\
Fluvial Processes & Alan Howard \\
\hline
\end{tabular}

priorities of the final landing sites to NASA HQ. The principal learning point is that there can be no substitute for extensive team discussion, so that everyone has their say, regardless of the outcome. In this case it was clear that there were two landing sites judged to be of distinctively high value to the Science Team: Eberswalde and Gale. These two sites were discussed additionally by the PSG, insuring that each PI would be comfortable with the operation of their instrument, and in the pursuit of the primary goals of the mission. In this forum, it was judged that both Eberswalde and Gale be presented to the NASA Associate Administrator for consideration, with a preference for Gale over Eberswalde. 
Table 7 Gale Ascent Team Working Group

\begin{tabular}{|c|c|}
\hline Name & Affiliation \\
\hline Matthew Golombek ${ }^{\mathrm{a}}$ & Jet Propulsion Laboratory \\
\hline Ken Herkenhoff ${ }^{\mathrm{a}}$ & U.S. Geological Survey, Flagstaff \\
\hline Ryan Anderson & Cornell University \\
\hline Paolo Belutta & Jet Propulsion Laboratory \\
\hline Timothy Parker & Jet Propulsion Laboratory \\
\hline Robert Sullivan & Cornell University \\
\hline Dawn Sumner & University of California, Davis \\
\hline
\end{tabular}

\subsection{Gale Ascent Team Working Group}

This Working Group (Table 7) was chartered for the purpose of assessing the traversability of the lowermost portion of Mount Sharp, specifically to those targets judged to be of high scientific interest: layered clays, layered sulfates, and the filled fractures.

Investigation of the Gale field site requires driving out of the ellipse to the south to access the high science value targets. A terrain and rover traversability map is shown in Fig. 9. As a result, the ellipse must be traversable to the south and the lower part of Mount Sharp must be accessible by the rover. Although there are no mobility concerns for most of the landing ellipse, a series of dark, fresh sand dunes that could be active (Hobbs et al. 2010) extend from the southern edge of the ellipse to the northeast. Examination of these dunes in HiRISE images and slope maps shows many of the dunes exceed the slope limit for driving on cohesionless material, but that there are a number of traversable troughs mostly swept clean of dark sand that cross the dune fields from north to south (Fig. 10). As a result, traversing to the south to exit the landing ellipse appears feasible.

South of the Gale ellipse a mineralogical stratigraphy has been identified in CRISM (Compact Reconnaissance Imaging Spectrometer for Mars) spectra that includes a topographically lowermost sulfate rich layer with an overlying clay-bearing layer that is overlain by more sulfates, with mixed clay and sulfate layers in between (Milliken et al. 2010). The team would like to be able to sample these strata to address the compelling science topics at this site. Examining the slope maps of the lower reaches of Mount Sharp and correlating with the hydrated mineral-bearing strata identified in CRISM shows that the lowermost sulfate layer is easily accessible south of the dune field. The boundary between the lowermost sulfates and the overlying clay-bearing unit occurs at the first steep slope, that has been called the "first fence" (Fig. 11). Between 10 and 20 paths have been identified through the "first fence," so access to the clay layer is also possible.

The mixed sulfate and clay strata above the clay layer begins above a second zone of steep slopes called the "second fence," which also has multiple traversable paths (5-10) through it, so the rover should be easily able to sample this unit as well (Fig. 11). After this unit, the topography of Mount Sharp steepens substantially and all travel is funneled into a number of relatively low slope pathways that weave among buttes and in steep sided canyons. There are over 10 possible pathways that cross the first steep line of mesas and buttes. Driving up any one of them would provide access to the overlying sulfate-bearing strata. Beyond the first line of steep mesas and buttes about 6 possible pathways could allow driving up to the uppermost light-toned unit that unconformably overlies the sulfate-bearing rocks. Ubiquitous steep slopes in this uppermost unit indicates it is probably not traversable within the area defined by current HiRISE coverage. A number of narrow potential choke points, that are 5-10 m wide, or require bedrock to reduce wheel slip in order to traverse 


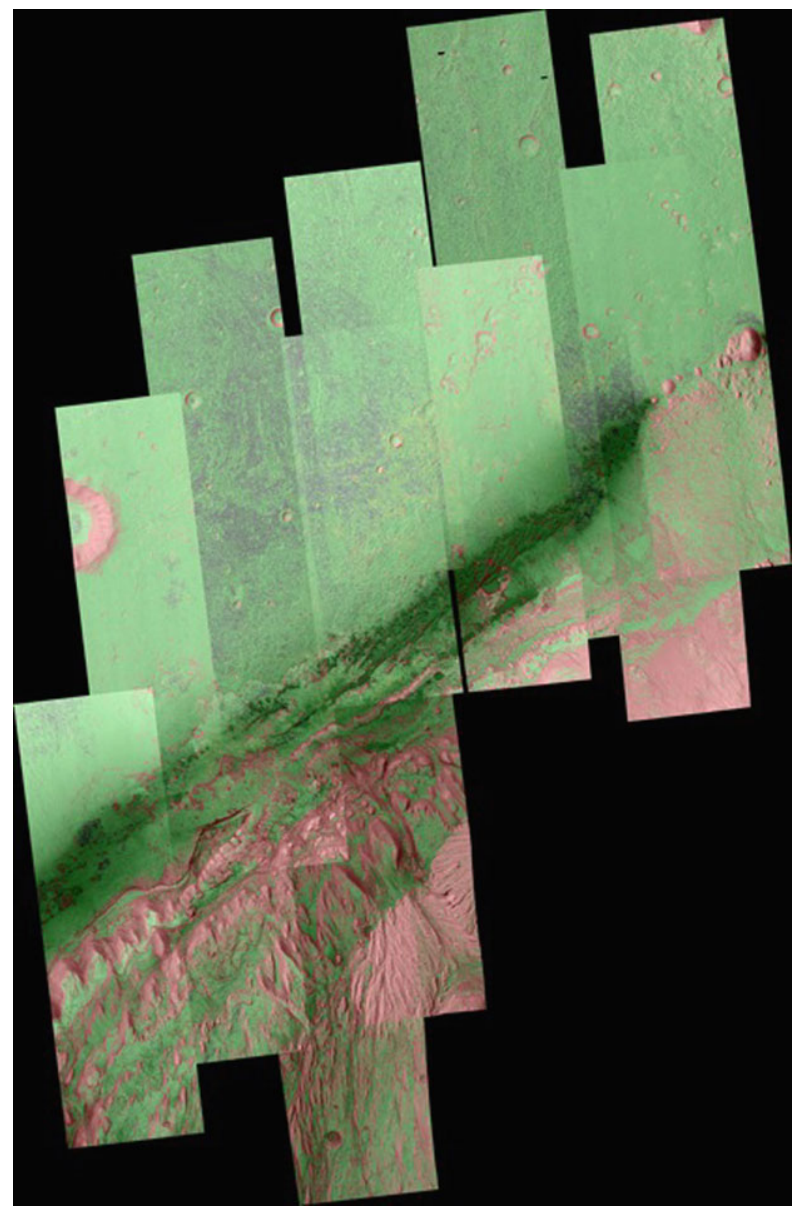

Fig. 9 Traversability map for Gale crater. See Sect. 3.7 (Navigation) for discussion of driving modes. Gray areas correspond to blind drive mode, blue areas to Autonav drive mode, green areas to $\mathrm{Vi-}$ sodom drive mode, yellow to Autonav + Visodom, and red areas are currently considered to not be traversable. Map shows most of the ellipse can be traversed in blind mode. Rougher areas to the east require driving using Visodom. The area to the south of the ellipse has zones of nontraversable terrain as well as areas requiring Visodom. To drive up the lower part of the mound requires driving in lower slope valleys and troughs with steeper walls. Not-traversable areas are shown in red in the mound, where driving is funneled into discrete drive paths. Image is $27.3 \mathrm{~km}$ by $39.4 \mathrm{~km}$. For scale, each image is $6 \mathrm{~km}$ wide. From left to right: ESP_012551_1750, ESP_024234_1755, PSP_009716_1755/PSP_009650_1755 (stereo pair), PSP_009294_1750/PSP_009149_1750 (bottom stereo pair), ESP_018854_1755/ESP_018920_1755 (top stereo pair), PSP_010639_1755/PSP_010573_1755 (stereo pair), ESP_018854_175, PSP_009571_1755/PSP_009505_1755 (stereo pair), ESP_024102_175N, ESP_0241102_1755, ESP_011417_1755/ESP_011562_1755 (stereo pair). HiRISE data courtesy of NASA/JPL/U. Arizona. Stereo DEM generation by Randy Kirk, USGS

slopes $>15^{\circ}$, have been identified along many of these pathways (Fig. 11). Given uncertainties in the stereo derived elevations and difficulties in uniquely identifying soil patches from layered rock along many of the paths suggests it may be difficult to be sure about the traversability of some of them. Nevertheless, the fact that multiple paths allow access to 

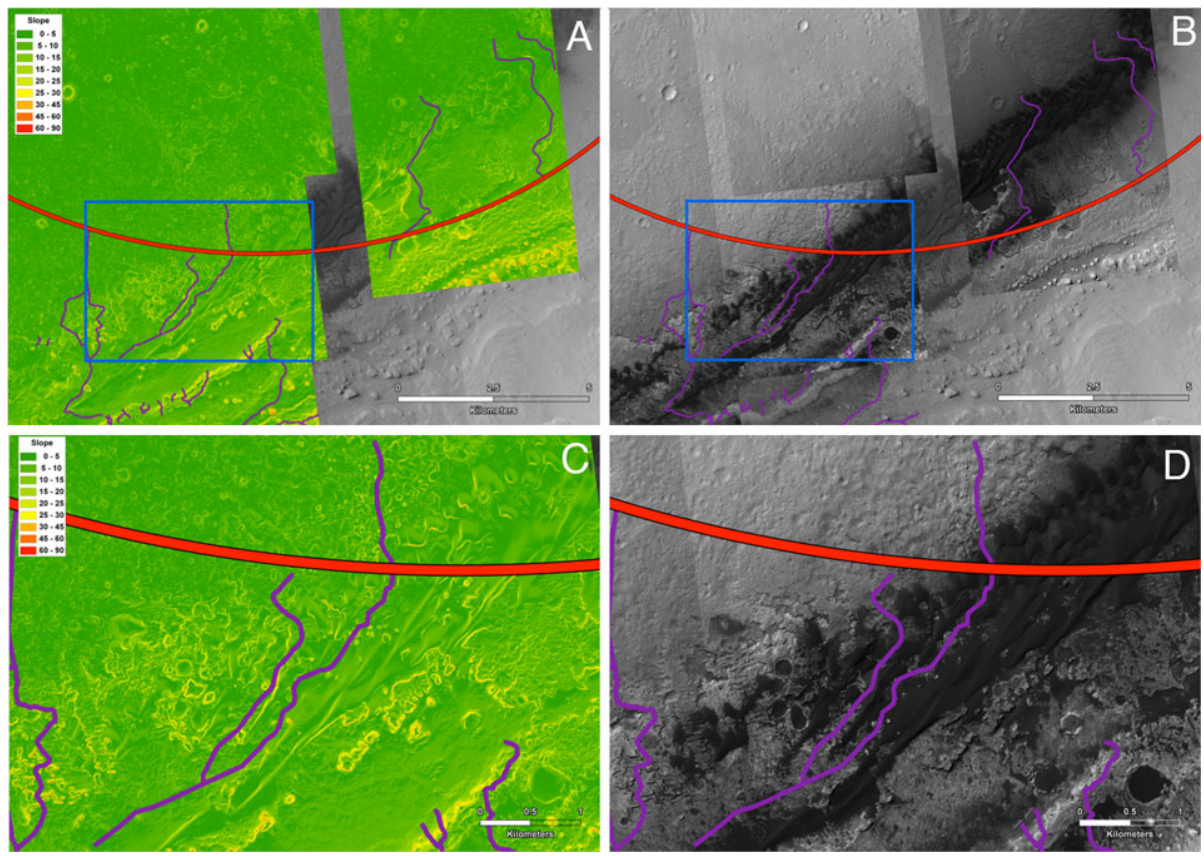

Fig. 10 Paths through eolian bedforms at base of Mount Sharp, localized at southern end of Gale ellipse (marked in red). One meter slope map (A) and corresponding HiRISE image (B) showing traversable paths (purple color) through bedforms. Blue rectangle outlines detail shown in $\mathbf{C}$ and $\mathbf{D}$. One meter slope map (C) and corresponding HiRISE image (D) show details of traverse paths. Paths are low relief and partially swept clear of sediment. HiRISE over CTX image. HiRISE images used: PSP_009716_1755/PSP_009650_1755 (stereo pair), ESP_018854_1755/ESP_018920_1755 (stereo pair), PSP_010639_1755/PSP_010573_1755 (stereo pair), PSP_009571_1755/PSP_009505_1755 (stereo pair). CTX image used: B07_012195_1750_NS_05S222W

all of the key mineral layers identified from orbit, indicates that the main science goals of sampling these layers can be accomplished by driving up Mount Sharp.

\section{The Curiosity Rover}

\subsection{Basic Description}

The core of the MSL flight system used in the surface mission is the rover, named Curiosity, which features an extensive scientific payload described briefly below, but in detail in other papers of this issue. Figure 12 indicates the location of some of the major components on the rover. A similar figure showing the location of the instruments (Fig. 19) is provided at the beginning of Sect. 4 .

Overall characteristics of Curiosity include a total mass of $899.2 \mathrm{~kg}, 2.8 \mathrm{~m}$ width, $3 \mathrm{~m}$ length (4.7 m long with robotic arm extended), $1.1 \mathrm{~m}$ top deck height, $2.2 \mathrm{~m}$ total height, and $75 \mathrm{~kg}$ instrument payload. The rover is a vehicle for remote operation on the Martian surface with the following capabilities:

- supports the science instrument payload investigations 


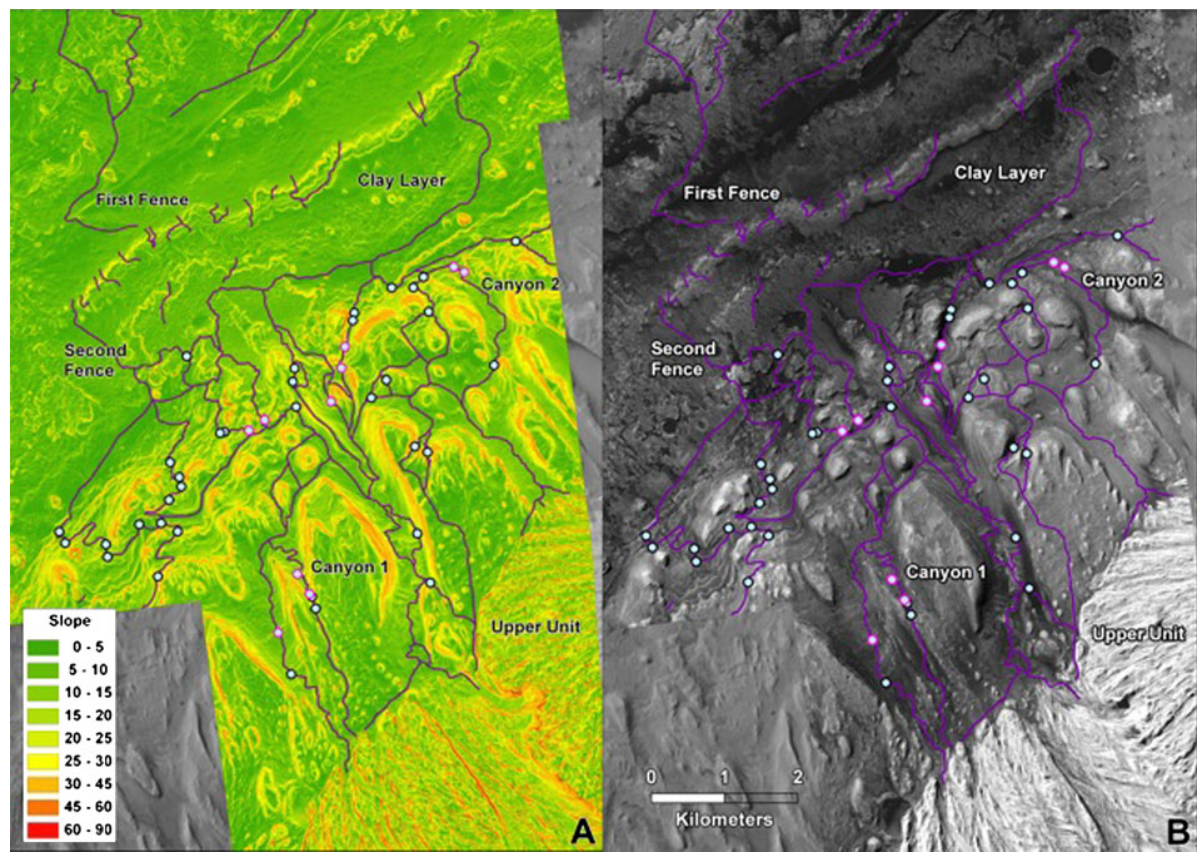

Fig. 11 Paths through the lower part of Mount Sharp. One meter slope map (A) and corresponding HiRISE image (B) showing traversable paths (in purple) through high science priority clay and sulfate deposits south of the Gale crater ellipse. Choke points where the traverse path must go through a narrow canyon are shown as dots. Blue dots are the locations where the slope along the traverse approaches the rover limit for the observed terrain type. Pink dots are where the traverse path is between 5-10 m wide; all others are $>10 \mathrm{~m}$ wide. HiRISE images over CTX image. HiRISE PSP_009294_1750/PSP_009149_1750 (stereo pair for slope map on east side) and PSP_019698_1750/PSP_019988_1750 (stereo pair for slope map on west side). CTX image used: B07_012195_1750_NS_05S222W

- can traverse up to 100 to 200 meters per sol, depending on the terrain

- provides high-speed computational capability and substantial data storage

- provides X-band for Direct-to-Earth (DTE) and Direct-from-Earth (DFE) telecommunications, and the ability to communicate via UHF with Mars Reconnaissance Orbiter and Mars Odyssey (which will store and relay data to the Earth)

In partnership with Disney-Pixar's animated film, WALL-E, NASA's Mars Public Engagement Program ran a rover-naming essay contest for K-12 students in the United States. Over 9,000 students sent in their suggestions. Twelve-year-old Clara Ma from Sunflower Elementary School in Lenexa, Kansas submitted the winning entry "Curiosity."

\subsection{Mobility}

Curiosity is a scaled version of the 6-wheel drive, 4-wheel steering, rocker bogie system from the Mars Exploration Rovers (MER) Spirit and Opportunity and the Mars Pathfinder Sojourner. Based on the center of mass, the vehicle is required to withstand a static tilt of at least $50^{\circ}$ in any direction without overturning. Fault protection will limit the rover from exceeding $30^{\circ}$ tilts while driving. The design of the rocker-bogie allows the wheels to move over objects approximately as large as the wheel diameter $(0.5 \mathrm{~m})$. Clearance under the rover's body on flat ground is $66 \mathrm{~cm}$. Each wheel has cleats and is independently actuated 


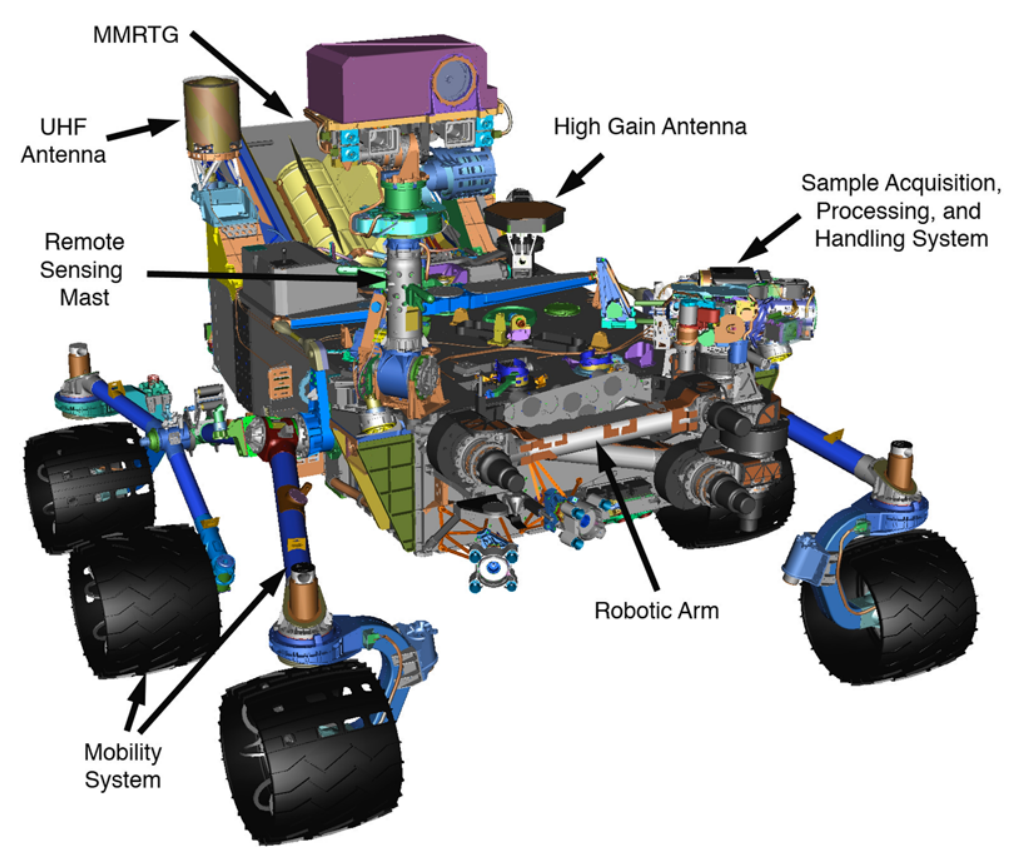

Fig. 12 Drawing of Curiosity indicating some of the major components. Robotic arm is stowed (folded up) in front of the rover

and geared, providing for driving in soft sand and climbing over rocks. Each front and rear wheel can be independently steered, allowing the vehicle to turn in place as well as execute arcing turns. The rover has a top speed on flat hard ground of $\sim 4 \mathrm{~cm} / \mathrm{s}$ but under autonomous control with hazard avoidance, the vehicle achieves an average speed of $\sim 1.5 \mathrm{~cm} / \mathrm{s}$. Further discussion of the driving modes occurs later in the section on navigation (Sect. 3.7).

Creation of terrain maps based on HiRISE and thermal inertia data were used to conduct simulated driving with Curiosity in the various modes described above. As applied to the Gale field site these simulations allowed assessment of the feasibility of driving upward through the strata which form the base of Mount Sharp, and through the eolian beforms which fringe its base (see Figs. 9, 10 and 11).

\subsection{Power}

Rover power is provided by the Multi-Mission Radioisotope Thermoelectric Generator (MMRTG; Fig. 12), which generates $\sim 110 \mathrm{~W}$ of electrical power at the start of the landed mission. Peak power demand from the rover activities easily exceeds this however, and the rover has two Li-Ion rechargeable $\sim 42$ amp-hour batteries to allow for all activities. The batteries are expected to go through multiple charge/discharge cycles per Sol, with minimum allowed state of charge of $\sim 40 \%$.

\subsection{Telecom}

The surface telecommunications system uses three antennas, two for X-Band DTE/DFE (Direct to/from Earth), and a UHF antenna for relay to an orbiting asset (Fig. 12). The X-band antennas are the rover Low Gain Antenna (LGA) and the High Gain Antenna (HGA). The 
Fig. 13 Remote Sensing Mast showing location of ChemCam, its associated Remote Warm Electronics Box (RWEB), and location of Mastcams and Navcams



HGA is used for either direct-to-Earth (DTE) or direct-from-Earth (DFE), while the LGA is used primarily for DFE. The basic telecom requirement for surface operations on the HGA is to transmit at least at 160 bits per second to a 34-meter Deep Space Network (DSN) antenna, or 800 bits per second to a 70-meter DSN antenna. In safe mode, commands from the Earth will be received via the LGA, which does not require pointing. Limited capability for communications exists via the LGA (15 bits per second uplink at max range). Current expectations are for the typical daily uplink of commands via the HGA, taking approximately 15 minutes for a total volume of 225 kilobits. The HGA sits on a 2 degree-of-freedom gimbal, with 5 degree system pointing accuracy (including rover attitude knowledge), and is 0.28 meters in diameter.

The primary data return path for surface operations is via the UHF relay system, using the Mars orbiting assets, Mars Odyssey and Mars Reconnaissance Orbiter (MRO). The Project intends for primary communications to go through MRO, with two passes a day primarily used to return data from the surface. The mission is designed to return a minimum of 250 megabits per sol using two UHF passes. Communications with Odyssey are subject to necessity and available energy. The UHF subsystem has a pair of redundant Electra-Lite radios. If for any reason DTE/DFE via X-Band is not possible, the UHF passes can be used to command the rover instead. A single quad-helix antenna called the RUHF is mounted to the rover deck and used for either of the radios.

\subsection{Remote Sensing Mast}

The Remote Sensing Mast (RSM), shown in Fig. 13 provides a tall geologist's eye-level view from the cameras mounted at the top, $\sim 2$ meters above the Martian surface. The RSM head includes the ChemCam, Mastcams, and Navcams, with the ChemCam sitting inside of the remote warm electronics box (R-WEB), a thermally controlled enclosure atop the mast. The RSM has the ability for azimuth and elevation control, and can slew at $5^{\circ}$ per second. The RSM allows for full $360^{\circ}\left( \pm 181^{\circ}\right)$ azimuth and $+91^{\circ}$ to $-87^{\circ}$ range of elevation range of motion. Mounted along the shaft of the mast are two booms for the REMS investigation.

\subsection{Engineering Cameras}

In addition to five science cameras described below in Sect. 4 of this paper, the MSL rover carries twelve engineering cameras (4 Navcams and 8 Hazcams), all of which share the same 
design as the MER engineering cameras described in Maki et al. (2003). The primary set of engineering cameras is a Navcam pair at the top of the mast (Fig. 13), a front Hazcam pair mounted on the front panel of the rover body (Fig. 12) and a rear Hazcam pair mounted on the back panel of the rover body. In contrast to MER, three pairs of the cameras on MSL provide redundant backups (an extra Navcam pair and an extra Front/Rear Hazcam pair). The redundant cameras are connected to the backup rover computer and are not expected to be used unless there is a problem with the primary rover computer and/or primary cameras. The engineering cameras are designed mainly to support operational activities such as rover navigation, localization, hazard detection, and robotic arm positioning. However, the operational distinction between science and engineering is only a convention; most downlinked Navcam/Hazcam image data will have value for both science and engineering.

The Navcams are mast-mounted stereo pairs of cameras (Fig. 13). Unlike MER, where much of the robotic arm workspace was blocked from view from the mast-mounted cameras, most of the robotic arm workspace for MSL will be viewable by the MSL Navcams, as well as the mast-mounted science cameras. The primary Navcam stereo pair is mounted near the top of the rover mast below the ChemCam mast unit, 1.99 meters above the ground when the rover is on hard flat terrain. The redundant Navcam pair is mounted $5 \mathrm{~cm}$ below the primary pair. The mast can point the camera pairs $360^{\circ}$ in azimuth and $+91^{\circ}$ to $-87^{\circ}$ range of elevation (up and down). The Navcams will be primarily used for navigation purposes and general site characterization, capable of providing $360^{\circ}$ panoramic image mosaics and targeted images of interest, including terrain not viewable by the Hazcams. The Navcam stereo pair is boresighted with the Mastcam science cameras and Chemcam science instrument, and Navcam images will often be used for science target selection and analysis.

The hazard-avoidance cameras (Hazcams) are mounted in stereo pairs; two pairs on the front panel of the rover body (Fig. 12) $68 \mathrm{~cm}$ above the ground when the rover is on hard flat terrain, and two pairs on the rear panel of the rover body $78 \mathrm{~cm}$ above the ground. Each Hazcam pair assembly includes two cameras mounted to achieve a stereo view and each Hazcam camera has a $124^{\circ} \times 124^{\circ}$ field of view. The Hazcams provide imaging primarily of the near field $(<5 \mathrm{~m})$ both in front of and behind the rover. These cameras will provide for onboard hazard detection using stereo data to build range maps. They also support science operations for selecting near field target and robotic arm operations.

The MSL engineering cameras are described in more detail in Maki et al. (2012, this issue).

\subsection{Navigation}

A very significant part of Curiosity's mission, given its overall goal of exploring and assessing a local region on Mars' surface, is its ability to "traverse" large distances. Like Spirit and Opportunity, Curiosity offers three primary modes of navigation. The first of these is the blind-drive, where rover planners have sufficient local imaging from the engineering cameras, to determine that a safe path exists, free of obstacles or hazards, and to command the rover to traverse some number of meters along that path. This length is usually limited by the visibility of distant terrain and ability to resolve hazards with the mast imagers. In this mode, wheel odometry is used to measure the distance traveled by the rover.

For a long traverse, the rover can select a path on its own by using hazard avoidance. Hazard avoidance requires the rover to stop frequently, on the order of a vehicle length, to acquire images with the Hazcams. The rover then analyzes the images for potential hazards, and selects a safe path. The algorithm on board has weights for path safety and how much the path corresponds to the desired direction of traverse. Visual odometry (aka "Visodom") consists of another image-based check of the drive. The rover stops at a given interval, roughly 
every 10 meters, to capture images orthogonal to the drive direction with the Navcam. It then compares the most recent image to an image acquired before the last segment of traverse, looking for similar features to determine how far it has gone. This mode is also called "slip-check" mode. This mode typically provides more accurate measurement of distance, as it is possible for the rover to slip, or turn wheels without making full progress-in fact the rover could potentially identify if it was stuck before aggravating the situation with further attempts to traverse.

The final drive mode utilizes both hazard avoidance and full-time visual odometry. The primary difference from the second mode described above is that the rover stops and performs the visual odometry analysis on the order of every half-vehicle length. This mode is useful when it is desired to perform a high precision approach of a target, and when the rover is traversing slippery or very steep terrain.

These modes differ significantly in the fraction of time spent moving versus computing. From a speed perspective, blind drives are fastest, followed by hazard avoidance with slip check, and then hazard avoidance with visual odometry. For comparison, typical rates on a flat sandy surface are $\sim 140 \mathrm{~m} / \mathrm{h}$ for blind drive, versus $\sim 45 \mathrm{~m} / \mathrm{h}$ for hazard avoidance, versus $\sim 20 \mathrm{~m} / \mathrm{h}$ for hazard avoidance with visual odometry.

In addition to providing mobility navigation on the surface of Mars, the surface attitude control system is responsible for maintaining an onboard estimate of the rover orientation, which is used both by ground analysts for interpreting the science and engineering data returned as well as onboard for the pointing of the High Gain Antenna. The rover's Inertial Measurement Unit is used to provide absolute tilt estimates relative to gravity as well as to propagate attitude during driving using gyros. Overall rover attitude knowledge (including heading) is determined from the position of the Sun using Navcams, similar to MER.

\subsection{Sample Acquisition, Processing, and Handling Subsystem}

The Sample Acquisition, Processing, and Handling (SA/SPaH) subsystem (Fig. 12) is responsible for the acquisition of rock and soil samples from the Martian surface and the processing of these samples into fine particles that are then distributed to the analytical science instruments, SAM and CheMin. The SA/SPaH subsystem is also responsible for the placement of the two contact instruments, APXS and MAHLI, on rock and soil targets. $\mathrm{SA} / \mathrm{SPaH}$ consists of a Robotic Arm (RA) and turret-mounted devices on the end of the arm, which include a drill, brush, soil scoop, sample processing device, and the mechanical and electrical interfaces to the two contact science instruments (Fig. 14). SA/SPaH also includes drill bit boxes, the Organic Check Material (OCM), and an observation tray, which are all mounted on the front of the rover, and inlet cover mechanisms that are placed over the SAM and CheMin solid sample inlet tubes on the rover top deck. Figure 15 shows the location of these $\mathrm{SA} / \mathrm{SPaH}$ components on the rover.

The Robot Arm (RA) is a 5 degree-of-freedom manipulator that is used to place and hold the turret-mounted devices and instruments on rock and soil targets, as well as manipulate the turret-mounted sample processing hardware. It has a mass of $70 \mathrm{~kg}$. When fully extended straight ahead in the rover forward drive direction, the center of the turret of the robotic arm is $1.9 \mathrm{~m}$ from the front of the rover body. At the end of the RA is the turret structure, which is $60 \mathrm{~cm}$ in diameter and has a mass of $30 \mathrm{~kg}$, on which 5 devices are mounted. Two of these devices are the science contact instruments APXS and MAHLI. The remaining three devices are associated with sample acquisition and sample preparation function: the Powder Acquisition Drill System (PADS), Dust Removal Tool (DRT), and the Collection and Handling for Interior Martian Rock Analysis (CHIMRA). 
Fig. 14 Diagram showing the turret-mounted devices on the end of the robotic arm: drill, brush, soil scoop, sample processing device (sieves, portioners), and the two contact science instruments, APXS and MAHLI. The devices are connected to the arm by the component shown in red on the underside of this drawing
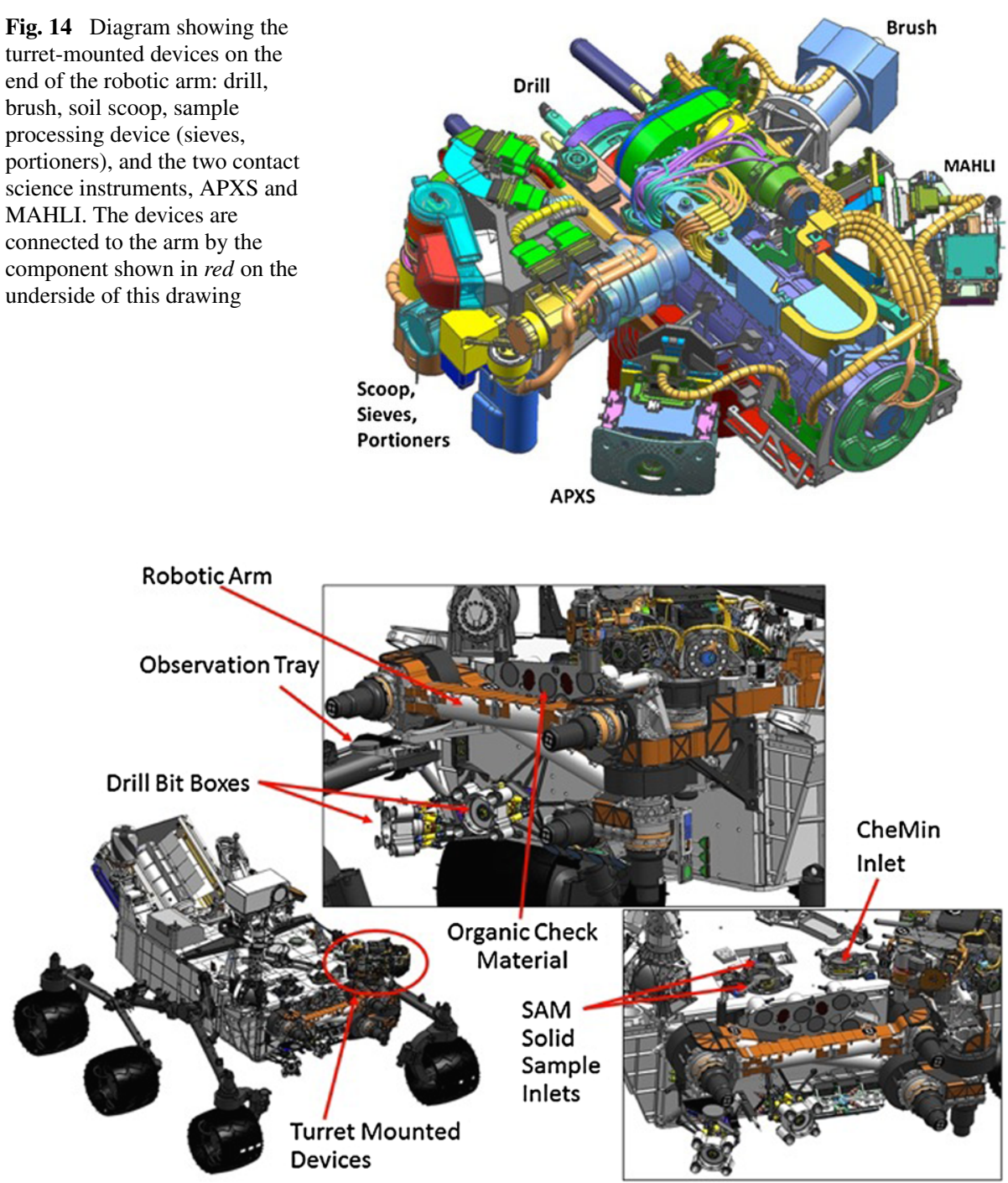

Fig. 15 Diagram showing the location of $\mathrm{SA} / \mathrm{SPaH}$ components on the rover, including bit boxes, organic check material, observation tray, and sample inlets

The PADS is responsible for acquiring powdered rock samples; the diameter of the hole in a rock after drilling is $1.6 \mathrm{~cm}$ in diameter and up to $5 \mathrm{~cm}$ deep. The powder travels up an auger in the drill and into a chamber with a transfer tube connection to the CHIMRA processing unit. Movement of the powder through CHIMRA is driven by gravity (by changing the position and orientation of the robotic arm) and vibration. To mitigate the risk of stuck or worn drill bits, the drill can disengage from the bit and SA/SPaH can reload one of the two spare bits located in containers called "bit boxes" on the front of the rover (Fig. 16).

Soil samples are acquired with CHIMRA's clam-shell scoop mechanism (Fig. 17), which can collect loose soil material from depths of up to $3.5 \mathrm{~cm}$, including the bottom of wheeldug trenches. The volume of a scooped soil sample is expected to be between 1 and $30 \mathrm{~cm}^{3}$. 
Fig. 16 Diagram of a portion of the front of the rover, showing location of the two bit boxes, observation tray above the bit boxes, and the five organic check material canisters

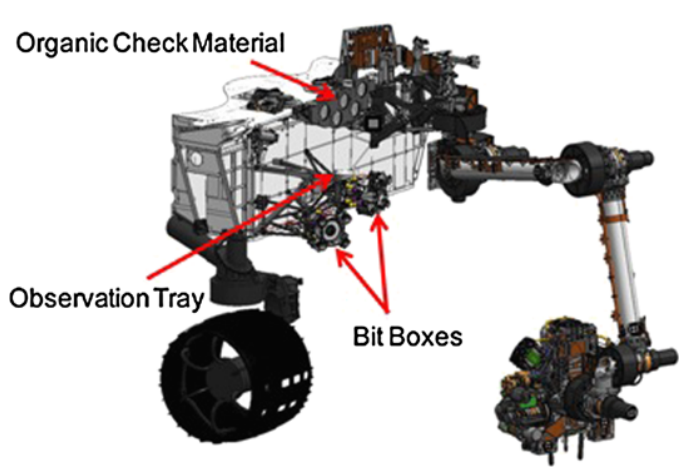

Fig. 17 Diagram of CHIMRA, showing the scoop in an open position (indicated here in red)



The CHIMRA sieves and portions the samples from the scoop and the drill for distribution to the analytical instruments, SAM and CheMin. Various chambers and labyrinths within the mechanism are used to sort and sieve the drilled rock powder or scooped soil material. The CHIMRA provides mechanisms for sieving particles to less than $150 \mu \mathrm{m}$, mixing the sieved samples, and portioning the samples for distribution to the SAM and CheMin instruments. The CHIMRA also provides the capability for sieving particles to less than $1 \mathrm{~mm}$ and portioning that material into an appropriate volume for distribution to the SAM instrument (45-130 $\mathrm{mm}^{3}$ per portion). Some cross-contamination between samples is expected due to fines that cling to the various surfaces of the SA/SPaH subsystem.

\subsubsection{Instrument Inlet Covers}

The SA/SPaH subsystem also provides covers that protect the SAM and CheMin solid sample inlets and sample funnels from being contaminated by particulates from the atmosphere or rover deck. These covers are mounted on top of each solid sample inlet (locations shown in Fig. 15) and are actuated with individual motors. During sample delivery, the instrument inlet cover is opened and the CHIMRA sample chamber is then positioned over top of the exposed solid sample inlet. Once the CHIMRA has dropped the sample into the solid sample inlet, the inlet cover is closed. The sample moves down through a funnel and into the instrument.

The MSL sample transfer chain is susceptible to wind effects during the drop off phase where a $2-5 \mathrm{~cm}$ vertical air gap must be crossed by the delivered portion as it comes out of CHIMRA and goes into the instrument funnels. In order to minimize the vulnerability of the portion during this drop off activity, a wind guard system hardware was added. This system 
Fig. 18 Flight Dust Removal Tool (DRT). The bristles extend $\sim 20 \mathrm{~mm}$ beyond the center post and the diameter of the brush area is $\sim 45 \mathrm{~mm}$

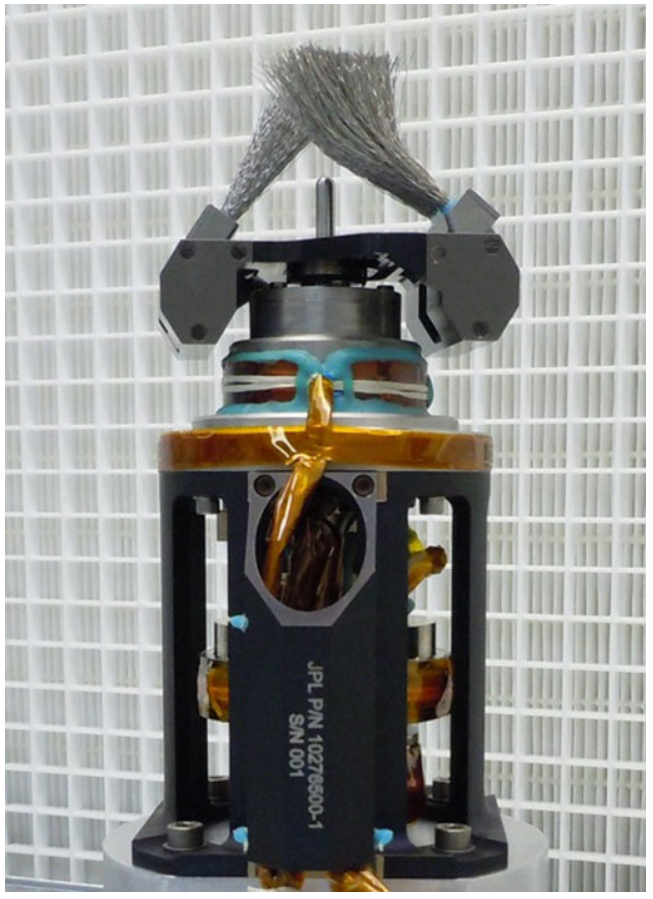

consists of a plate, mounted to CHIMRA, that presses down against a skirt that surrounds the SAM and CheMin instrument funnels. The skirt element is hard metal in a horse shoe shape, and the tutu consists of a flexible fabric.

\subsubsection{Dust Removal Tool}

The Dust Removal Tool (DRT) is mounted to the turret of the robotic arm and can be used to remove dust and loose material off of rock surfaces by clearing it away with stainless steel wire brushes. The design of the DRT (Fig. 18) is different from the Rock Abrasion Tool brushes on the Spirit and Opportunity rovers, but it is expected to have a similarly effective dust removal capability. A single actuator mechanism rotates the brushes and relies on the robotic arm to position it at a desired standoff distance from a target surface. The area cleared with the DRT has a minimum circular area of $45 \mathrm{~mm}$ diameter. The DRT is also expected to be used to clear off loose material from the observation tray.

The SA/SPaH subsystem is described in more detail in Anderson et al. (2012, this issue).

\subsection{Observation Tray and Sample Testing Tools}

A science observation tray is provided to allow processed samples delivered by CHIMRA to be observed by APXS and MAHLI. The science observation tray is a simple round, flat titanium metal tray $7.5 \mathrm{~cm}$ in diameter, mounted on the front of the rover (Fig. 16). Soil and rock samples that have passed through the $150 \mu \mathrm{m}$ sieve of CHIMRA can be deposited on the tray, observed by the APXS and MAHLI, and subsequently removed from the tray by the DRT brushes. After delivering sieved material (soil or rock) to SAM and/or CheMin, the remainder of the sieved material can be analyzed in this way. This tray and the sample testing tools next to it are viewable by the Navcam and Mastcam. 
When there are concerns about end-to-end sample delivery, additional sample testing tools installed next to the Science Observation Tray, along with imaging, could be used for qualitative characterization of the physical behavior of processed drill powder or scooped soil before attempting delivery to the instruments. Next to the science observation tray is an engineering observation tray that is made out of aluminum alloy with a checkerboard anodized grid pattern on it to assist with estimation of areal extent of deposited material. Next to the Observation trays, a CheMin-surrogate funnel is mounted that is the same design as the CheMin inlet funnel but from which a $62^{\circ}$ segment has been removed to permit sideviewing into the funnel by MAHLI. A capture plate with concave-up geometry is present underneath to catch any material that falls out. This surrogate funnel can be used (along with imaging) to assess whether a sample is likely to clog the instrument funnels before attempting a delivery, and might indicate relative stickiness of the material by how far it slides down the capture plate.

The rover has two spring loaded "pokers" which are intended as options for clearing potential clogs to the portion tube inside CHIMRA that deliver the sieved $150 \mu \mathrm{m}$ portion to the instruments. Each poker has identical geometry and springs, but one is mounted vertically (this is the primary poker) and one is mounted horizontally (the secondary poker). The poker tip is $2.15 \mathrm{~mm}$ in diameter and is designed to fit inside the portion tube-a tapered cylinder. The tube widens from $3 \mathrm{~mm}$ in diameter on the entrance to $4 \mathrm{~mm}$ at its exit, where the sample then moves on to the instruments. The operational scenario for the poker utilizes the robotic arm plunging the CHIMRA portion tube down onto the poker in a rastor scan pattern.

\subsection{Organic Check Material}

Steps have been taken to ensure that the SAM measurements of soil and rocks on Mars do not contain terrestrial contaminants above the SAM detection levels (ten Kate et al. 2008). However, it is likely that a slight amount of terrestrial contamination may be present despite our best efforts. To assess the characteristics of organic contamination at five different times in the mission, five bricks of Organic Check Material (OCM) mounted in canisters on the front of the rover (Fig. 16) will be available for end-to-end sample handling tests on Mars. Each OCM brick can be drilled, sieved and portioned in CHIMRA, and delivered to SAM (and optionally also to CheMin), with the OCM drilled powder following the same pathway as for drilled Martian rocks. Each brick is made of porous amorphous silicon dioxide ceramic with $30 \%$ interconnected porosity. The bricks are doped with a low concentration of 3-fluorophenanthrene and 1-fluoronapthalene, which are synthetic organic compounds not found in nature on Earth and not expected on Mars. Each of the bricks is sealed in its own canister and under vacuum until it is drilled into on Mars. Although the OCM material is $\mathrm{X}$-ray amorphous, it could be used to check the level of end-to-end cross contamination on Mars from sample to sample in CheMin, by looking for residual XRD pattern features or residual XRF element peaks (residual from previously acquired samples) in an OCM-filled sample cell.

See Conrad et al. (2012, this issue) for further description of the organic check material.

\section{Science Instrument Investigations}

Figure 19 shows the location of the 10 science instruments on the rover. There are four main types of instruments, the contact science instruments APXS (Alpha-Particle X-ray Spectrometer) and MAHLI (Mars Hand Lens Imager) on the turret of the robotic arm; the remote 


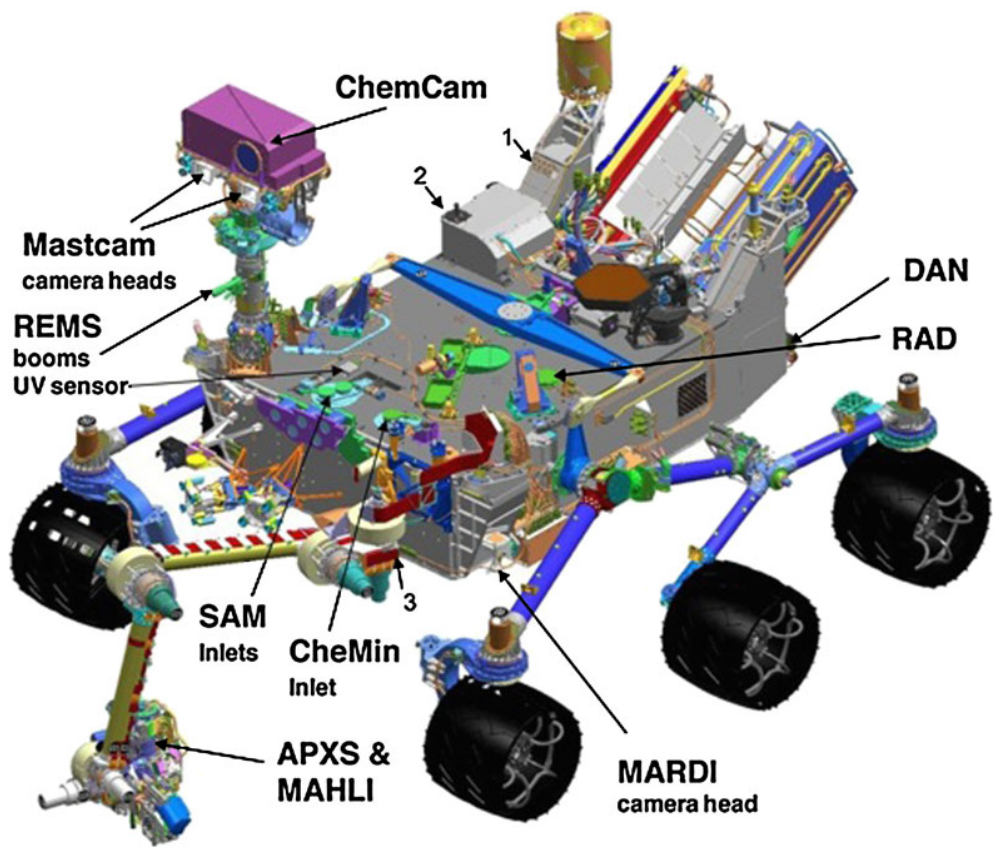

Fig. 19 Location of the instruments on the MSL rover. CheMin and SAM are inside the rover chassis. APXS and MAHLI are mounted on the turret on the end of the robotic arm. Their locations on the turret are shown in Fig. 14. An arrow points to the DAN detector and electronics. The DAN pulsing neutron generator is on the opposite mirror side of the rear of the rover. The location of calibration targets is shown for ChemCam (1) and Mastcam (2). The calibration targets for APXS and MAHLI (3) are located on a side of the robotic arm's azimuth actuator housing, hidden from view in this diagram

sensing instruments ChemCam (Laser-Induced Remote Sensing for Chemistry with its own remote Micro-Imager) and Mastcam (Mast Cameras); the environmental instruments DAN (Dynamic Albedo of Neutrons), MARDI (Mars Descent Imager), RAD (Radiation Assessment Detector), and REMS (Rover Environmental Monitoring Station); and the analytical laboratory instruments CheMin (Chemistry and Mineralogy) and SAM (Sample Analysis at Mars).

\subsection{APXS (Alpha-Particle X-ray Spectrometer)}

The main objective of the APXS (Alpha-Particle X-ray Spectrometer) instrument is to characterize the geological context of the rover surroundings and to investigate the processes that formed the rocks and soils. The high precision and low detection limits, especially for salt forming elements like $\mathrm{S}, \mathrm{Cl}$, and $\mathrm{Br}$, allow identification of local anomalies and will help inform selection of samples for further analysis by ChemMin and SAM. Samples placed on the Observation Tray will allow the APXS to provide additional characterization. Preparation of rock surfaces using the Dust Removal Tool will allow in-situ APXS investigations of thin alteration rinds or near-surface layers or veins which cannot be collected by the drill for the analytical instruments. Another important aspect of the APXS investigation will be to relate the chemical composition of rocks and soils to what has been found by the previous landed missions, which used similar X-ray spectroscopy methods. The APXS signal stems from the topmost micrometers for low $z$ elements like Na to $\mathrm{Si}$ and some $20 \mathrm{~s}$ of micrometers 
Fig. 20 Flight model APXS hardware: sensor head (right), electronics unit (left), and calibration target (middle front)

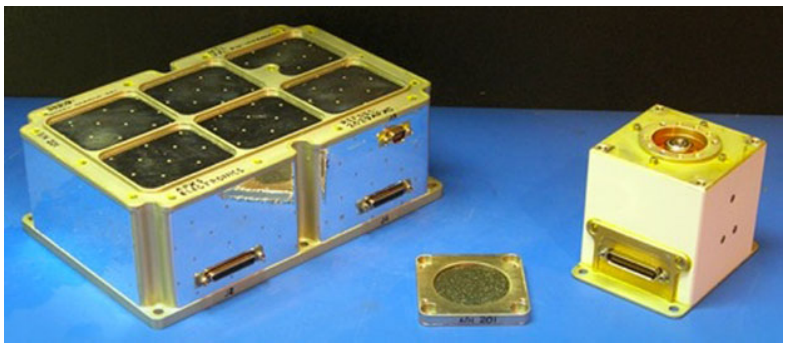

for heavier elements like Fe, mainly determined by the range of the characteristic elemental $\mathrm{x}$-ray radiation. Therefore preparation of rock surfaces using the dust removal tool will be helpful in order to study the outermost composition of rocks. Furthermore, it is planned to investigate the drill hole and the powder generated during drilling to get another APXS reading of the bulk chemistry of the rock interior.

The APXS for MSL is an improved version of the APXS that flew successfully on Pathfinder and the Mars Exploration Rovers Spirit and Opportunity (Rieder et al. 1997, 2003; Gellert et al. 2006). The MSL APXS takes advantage of a combination of the wellestablished terrestrial standard methods Particle-Induced X-ray Emission (PIXE) and X-ray fluorescence (XRF) to determine elemental chemistry. It uses curium-244 sources for X-ray spectroscopy to determine the abundance of major elements down to trace elements from sodium to bromine and beyond. With a half-life of 18 years for curium-244 there will be no discernible intensity loss during any extended mission.

The instrument (Fig. 20) consists of a main electronics unit in the rover's body and a sensor head mounted on the robotic arm. Measurements are taken by deploying the sensor head towards a desired sample, placing the sensor head in contact or hovering typically less than $2 \mathrm{~cm}$ away, and measuring the emitted X-ray spectrum for 15 minutes to 3 hours without the need of further interaction by the rover.

The MSL APXS has approximately 3 times the sensitivity for low $Z$ (atomic number) elements and approximately 6 times for higher $Z$ elements than the MER APXS. A full analysis with detection limits of $100 \mathrm{ppm}$ for $\mathrm{Ni}$ and $\sim 20 \mathrm{ppm}$ for $\mathrm{Br}$ now requires 3 hours, while "quick look" analysis for major and minor elements at $\sim 0.5 \%$ abundance, such as $\mathrm{Na}, \mathrm{Mg}, \mathrm{Al}, \mathrm{Si}, \mathrm{Ca}, \mathrm{Fe}$, or $\mathrm{S}$, can be done in 10 minutes or less. The sampled area is about $1.7 \mathrm{~cm}$ in diameter when the instrument is in contact with the sample. The APXS will be fully calibrated using standard geological samples in the laboratory. An onboard basaltic rock slab, surrounded by a nickel plate, will be used periodically to check the performance and calibration of the instrument.

Further description of the APXS instrument and science investigation can found in Campbell et al. (2012, this issue).

\subsection{ChemCam (Laser-Induced Remote Sensing for Chemistry and Micro-imaging)}

The ChemCam instrument package consists of a Laser-Induced Breakdown Spectrometer (LIBS) coupled to a telescopic camera known as the Remote Micro-Imager (RMI) (see Fig. 21). The LIBS provides elemental compositions, while the RMI places the LIBS analyses in their geologic context. Both instruments will help determine which rock and soil targets within the vicinity of the rover are of sufficient interest to use the contact and analytical laboratory instruments for further characterization. ChemCam can analyze a much larger number of samples than can be studied with the contact and analytical laboratory instruments. For example, the ChemCam team anticipates making daily analyses of the soil 
Fig. 21 Flight model ChemCam instrument. The mast-unit (top) is comprised of the laser, imager, telescope, and focus laser. The body-unit (bottom) includes an optical demultiplexer, three spectrometers, the central processing unit and a thermo-electric cooler
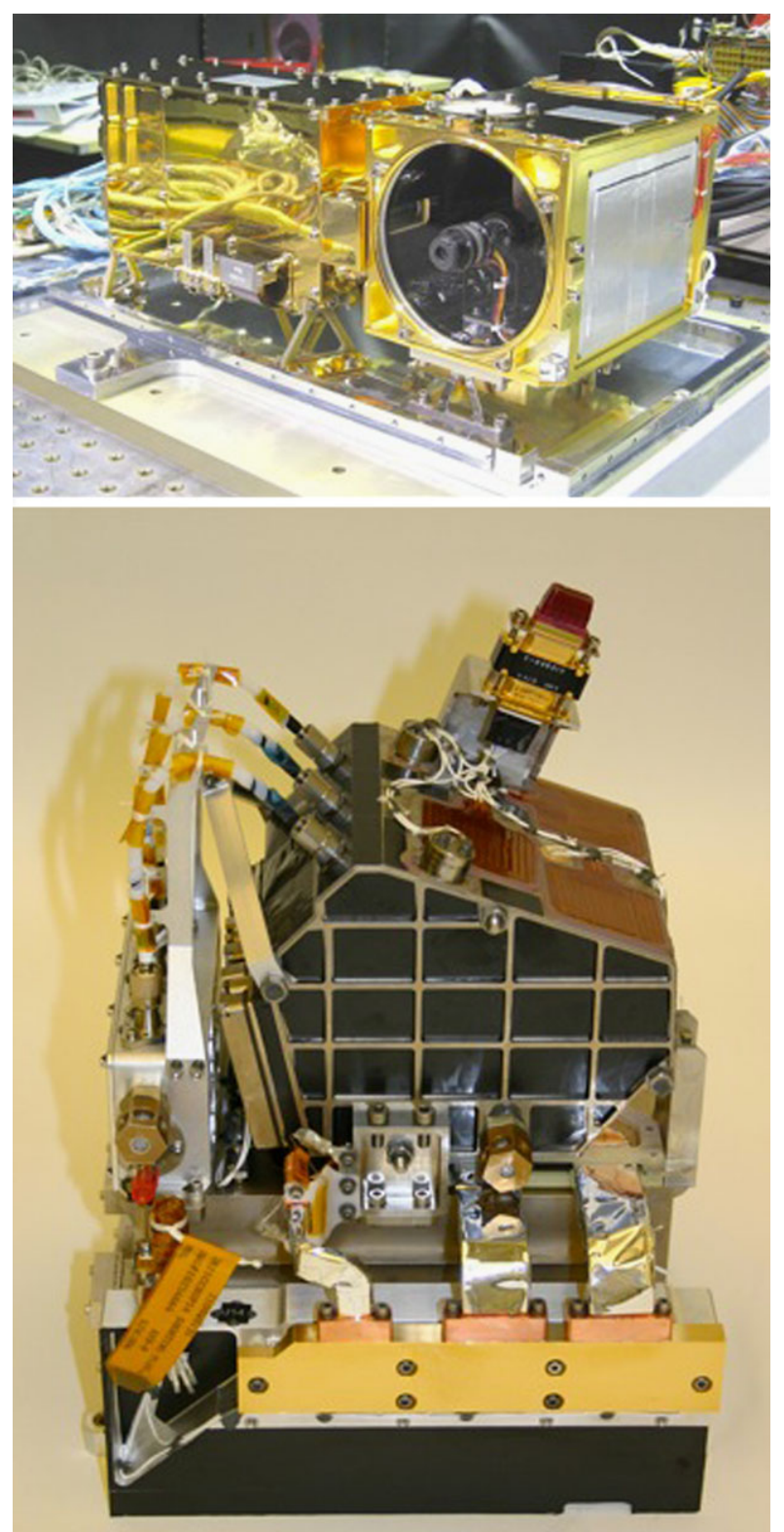

at the rover location to understand spatial variations in soil composition. Furthermore, it can provide valuable analyses of samples that are inaccessible to other instruments, such as vertical outcrops where LIBS can target individual strata using its submillimeter beam diameter. ChemCam uses multiple laser pulses to clean dust off of rock samples, providing uncontaminated remote observations, and it can also remotely analyze or profile through weathering rinds or surface coatings.

The LIBS instrument uses powerful laser pulses (Fig. 21, top), focused on a small spot on target rock and soil samples within $7 \mathrm{~m}$ of the rover, to ablate atoms and ions in electronically 
excited states from which they decay, producing light-emitting plasma. The power density needed for LIBS is $>10 \mathrm{MW} / \mathrm{mm}^{2}$, which is produced on a spot in the range of 0.2 to $0.6 \mathrm{~mm}$ diameter using focused, $\sim 14 \mathrm{~mJ}$ laser pulses of 5 nanoseconds duration. The plasma light is collected by a $110 \mathrm{~mm}$ diameter telescope and focused onto the end of a fiber optic cable. The fiber carries the light to three dispersive spectrometers (Fig. 21, bottom) that record the spectra over a range of $240-850 \mathrm{~nm}$ at spectral resolutions from 0.15 to $0.60 \mathrm{~nm}$ in 6144 channels. The spectra consist of emission lines of elements present in the samples. Typical rock and soil analyses yield detectable quantities of $\mathrm{Na}, \mathrm{Mg}, \mathrm{Al}, \mathrm{Si}, \mathrm{Ca}, \mathrm{K}, \mathrm{Ti}, \mathrm{Mn}, \mathrm{Fe}, \mathrm{H}$, $\mathrm{C}, \mathrm{O}, \mathrm{Li}, \mathrm{Sr}$, and $\mathrm{Ba}$. Other elements often seen in soils and rocks on Earth include $\mathrm{S}, \mathrm{N}, \mathrm{P}$, $\mathrm{Be}, \mathrm{Ni}, \mathrm{Zr}, \mathrm{Zn}, \mathrm{Cu}, \mathrm{Rb}$, and Cs. ChemCam LIBS is particularly sensitive to the alkali and alkaline earth elements, so it is expected to provide unique information, for example, on $\mathrm{Li}$, $\mathrm{Be}, \mathrm{Rb}, \mathrm{Sr}, \mathrm{Ba}$. Using the hydrogen emission line it will also supply important information on the hydration states of the samples it interrogates. It is anticipated that each analysis will use 50-75 laser pulses to achieve the desired $\pm 10 \%$ relative accuracy for major elements at $7 \mathrm{~m}$ distance.

The RMI is intended as a context imager for the LIBS, though unlike LIBS, it has no restrictions on the distance to the targets it images. It images through the same telescope as the LIBS (Fig. 21). The RMI detector is a $1024 \times 1024$ pixel CCD. The RMI can clearly distinguish the submillimeter LIBS spot on a metal plate at any distance within range of the LIBS. LIBS spots on rocks are more difficult to distinguish, but their locations on the images will be known from the pixel mapping, so the context of the LIBS spot can be determined.

The onboard rover calibration targets for LIBS consist of natural and synthetic volcanic glasses (Fabré et al. 2009, 2010) and ceramics consisting of mixtures of smectite and kaolinite with anhydrite and basalt to simulate Martian sedimentary samples (Vaniman et al. 2012). Also included is a graphite disk for carbon identification and a titanium plate for general use.

Additional information about ChemCam can be found in Wiens et al. (2012, this issue) and Maurice et al. (2012, this issue).

\subsection{CheMin (Chemistry and Mineralogy)}

An important science goal of the MSL mission is to identify and characterize past or present habitable environments as recorded in sediments and rocks. CheMin ("Chemistry and Mineralogy") is a definitive mineralogy instrument that will identify and quantify the minerals present in rocks and soil delivered to it by the SA/SPaH system. By determining the mineralogy of rocks and soils, CheMin will assess the involvement of water in their formation, deposition, or alteration. CheMin can identify and quantify minerals above its detection limits in complex natural samples such as basalts, multicomponent evaporite systems, and soils.

CheMin is a powder X-ray Diffraction (XRD) instrument that also has X-ray Fluorescence (XRF) capabilities (Fig. 22). CheMin is part of the Analytical Laboratory of the MSL rover, which is located inside the main body of the rover. CheMin will analyze as many as 74 samples delivered by the $\mathrm{SA} / \mathrm{SPaH}$ system during the nominal prime mission, but is capable of analyzing many more because its sample cells can be reused for additional analyses. CheMin utilizes a microfocus cobalt X-ray source, a transmission sample cell, and an energy-discriminating X-ray sensitive CCD to produce simultaneous 2-D X-ray diffraction patterns and energy-dispersive histograms from powdered samples. Raw CCD frames are processed into data products onboard the rover to reduce the data volume. Each analysis may take up to 10 hours of analysis time, spread out over two or more Martian nights, although some samples may provide acceptable results in a single sol. CheMin measurements are obtained during nighttime hours on Mars for several reasons the most important of 


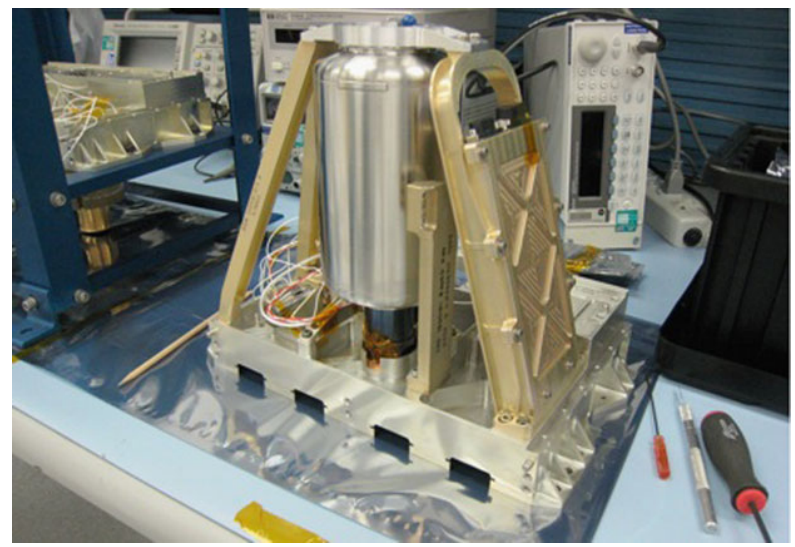

Fig. 22 CheMin DM instrument. The fully assembled instrument is $30 \times 30 \times 30 \mathrm{~cm}^{3}$ and has a mass of $10 \mathrm{~kg}$. In this view, in which the instrument enclosure and primary electronics box are removed, CheMin is inverted and resting on its "alignment bench." The alignment bench fixes the distances between the X-ray tube, sample wheel and CCD assembly, which must remain constant within a few tens of microns during a sample analysis. Seen are the alignment bench, the high voltage power supply controller assembly, the X-ray Source, and the Sample Wheel Dust Shroud

which is that RAMP (Rover Avionics Mounting Platform) temperatures are lowest at night, and since the RAMP is where CheMin's X-ray Source (XRS) and Charge-Coupled Device (CCD) cryo-cooler deposit their waste heat, RAMP temperatures dictate the operating temperatures of CheMin's X-ray source and CCD. Under CheMin's operational constraints, the RAMP must be below $+20{ }^{\circ} \mathrm{C}$ for the X-ray source to be energized, and this will occur principally at night.

In operation, a collimated X-ray beam from the X-ray tube is directed through a transmission sample cell containing powdered material prepared and delivered by the SA/SPaH system. An X-ray sensitive CCD imager is positioned on the opposite side of the sample from the source and directly detects X-rays diffracted or fluoresced by the sample. Both crystalline and amorphous materials can be analyzed in this fashion.

The CheMin instrument should be able to detect individual minerals in complex mixtures that are present at the $3 \%$ level and above. For minerals that are present in concentrations of $12 \%$ and above (4 times the minimum detection level), the goal is for CheMin to be able to state the absolute amount present $\pm 15 \%$.

For calibration, five permanent cells will be loaded with calibration standards. Three of these cells will be loaded with single minerals or a synthetic ceramic and two will be loaded with differing quartz/beryl mixtures. In use on Mars, two standards will be analyzed as soon as possible after landing. The standards to be analyzed are pure amphibole (for energy dispersive histogram calibration) and $97 \%$ beryl (for XRD calibration). Subsequent calibrations will be performed using one or more of the permanent standards on a nominal schedule of once every 40 sols.

Further description of the CheMin instrument can be found in Blake et al. (2012, this issue).

\subsection{DAN (Dynamic Albedo of Neutrons)}

The Dynamic Albedo of Neutrons (DAN) is an active/passive neutron spectrometer that measures the abundance and depth distribution of $\mathrm{H}$ - and $\mathrm{OH}$-bearing materials (e.g., adsorbed water, hydrated minerals) to a shallow depth $(\sim 1 \mathrm{~m})$ of Mars' subsurface along the 

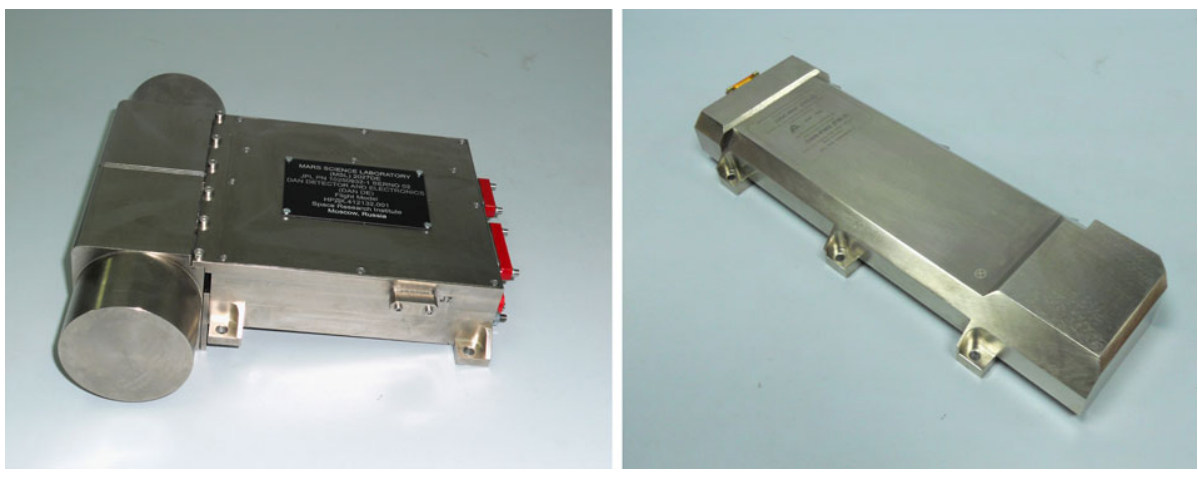

Fig. 23 Flight model of DAN instrument. Detector element (left) and pulse neutron generator (right)

path of the MSL rover. In active mode, DAN measures the time decay curve (the "dynamic albedo") of the neutron flux from the subsurface induced by its pulsing $14 \mathrm{MeV}$ neutron source (Fig. 23).

The science objectives of the DAN instrument are as follows: (1) Detect and provide a quantitative estimation of the hydrogen in the subsurface throughout the surface mission; (2) Investigate the upper $<0.5 \mathrm{~m}$ of the subsurface and determine the possible layering structure, via modeling, of hydrogen-bearing materials in the subsurface; (3) Track the variability of hydrogen content in the upper soil layer $(\sim 1 \mathrm{~m})$ during the mission by periodic analysis; and (4) Track the variability of neutron radiation background (neutrons with energy $<100 \mathrm{keV}$ ) during the mission by periodic analysis.

The DAN instrument is expected to be used during rover traverses (e.g., during short stops at $\sim 1 \mathrm{~m}$ intervals) and while the rover is parked. Short-duration $(<2 \mathrm{~min})$ measurements will provide a rough estimate of the water-equivalent hydrogen distribution with an accuracy of $\sim 1 \%$ by weight. Longer-duration $(\sim 30 \mathrm{~min})$ measurements are necessary to derive the vertical distribution of water-equivalent hydrogen with an accuracy of $0.1-0.3 \%$ by weight. A detailed description of the DAN instrument and scientific investigation can be found in Litvak et al. (2008), and in Litvak et al. (this issue). The instrument is contributed by the Federal Space Agency of Russia.

\subsection{MAHLI (Mars Hand Lens Imager)}

The Mars Hand Lens Imager (MAHLI) is a focusable color camera located on the turret at the end of the MSL robotic arm. The primary objective of the MAHLI investigation is to acquire images at, but not limited to, hand lens scale, which will facilitate the interpretation of the petrography and mineralogy of rocks and regolith fines at Gale crater. Additionally, images from MAHLI will be used to help select materials to be sampled or examined by the other instruments (particularly APXS, CheMin, and SAM) and document the sampled or examined targets and collected materials. Other applications of MAHLI include, but are not limited to, night imaging; searching for fluorescent materials using the UV LEDs; observing seasonal frost; monitoring overnight changes in frost; drill hole imaging; acquiring scientific video or public outreach sequences; rover problem diagnosis; and rover self portraits.

The MAHLI instrument consists of three major parts: a camera head (Fig. 24), a Digital Electronics Assembly (DEA) and a calibration target. The DEA is located within the rover body, the camera head is mounted on the turret at the end of the rover's robotic arm, and the calibration target also is mounted on the robotic arm. 
Fig. 24 Flight MAHLI camera head. The camera head consists of three functional elements: an optomechanical assembly, a focal plane assembly, and the camera head electronics assembly. The pocket knife is $88.9 \mathrm{~mm}$ long

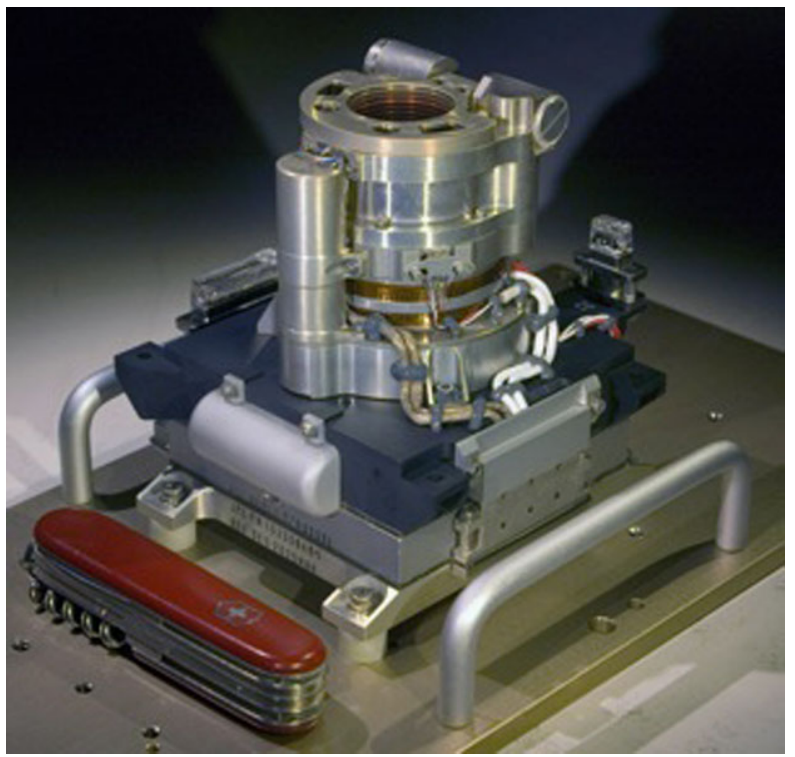

The instrument acquires images of up to 1600 by 1200 pixels with a color quality equivalent to that of consumer digital cameras. The spectral range for MAHLI images is 380$680 \mathrm{~nm}$. MAHLI images can be acquired at working distances between $21 \mathrm{~mm}$ and infinity, permitting acquisition of closeup views with a pixel scale/spatial resolution as high as $14 \mu \mathrm{m}$ per pixel, as well as selection of context views at greater working distances. The depth of field varies as a function of working distance, with the highest resolution MAHLI views having a depth of field of about $1.6 \mathrm{~mm}$; at the pixel scale of MER MI images ( $\sim 30 \mu \mathrm{m} / \mathrm{pixel})$, the depth of field is about $2 \mathrm{~mm}$.

The MAHLI includes two sets of two white light LEDs to permit nighttime imaging. Each pair can be independently commanded. MAHLI also has two ultraviolet (365 nm) LEDs to look for materials that fluoresce under longwave UV illumination. The UV LEDs are included on an exploratory, "best efforts" basis and are not a calibrated investigative tool.

The MAHLI instrument is discussed in detail in Edgett et al. (2012, this issue).

\subsection{MARDI (Mars Descent Imager)}

The Mars Descent Imager (MARDI) is a fixed-focus color camera mounted even with the bottom of the rover chassis (Fig. 25). MARDI's primary objective is to determine where exactly the vehicle has landed and to provide a geologic and engineering-geologic framework of the landing site for early operations.

The camera will take 1648 by 1200 pixel images at $\sim 4$ frames per second throughout the period of time between heatshield separation and touchdown plus a few seconds before heatshield deployment and after landing (a period of about two minutes). MARDI provides in-focus pixel scales that range from $1.5 \mathrm{~m}$ at $2 \mathrm{~km}$ altitude to $1.5 \mathrm{~mm}$ at $2 \mathrm{~m}$ altitude, and cover between 2.4 by $1.8 \mathrm{~km}$ and 2.4 by $1.8 \mathrm{~m}$ at these respective altitudes.

Although not originally proposed to be used after landing, once the rover is on the ground, MARDI can acquire images of a $0.8 \times 0.6 \mathrm{~m}$ section of the surface (although somewhat out of focus at resolution of about $3 \mathrm{~mm}$ ). Such use will be undertaken on a best efforts basis.

See Malin et al. (in preparation) for additional description of the MARDI instrument. 

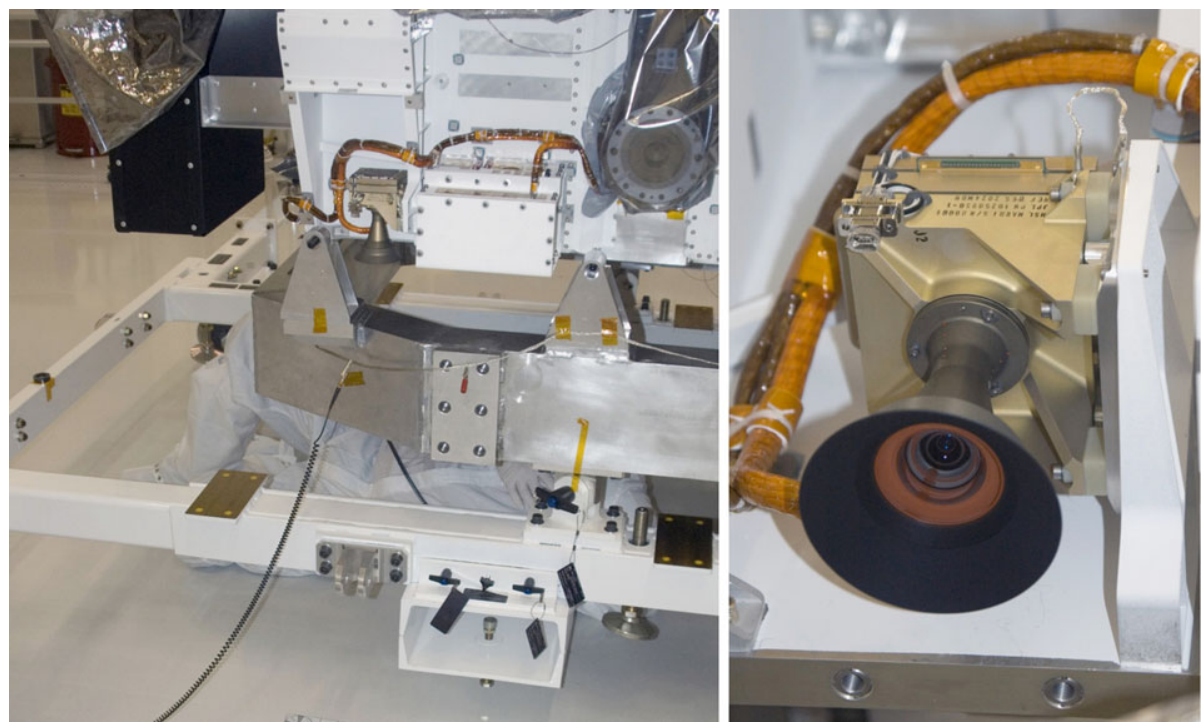

Fig. 25 Flight model of the MARDI camera. MARDI is mounted even with the bottom of the rover chasis, facing downward, and will operate from the time of heat shield separation until just after landing

\subsection{Mastcam (Mast Camera)}

The Mast Camera is a two-instrument suite of imaging systems mounted on the MSL rover's Remote Sensing Mast (RSM), with the boresight $\sim 1.97 \mathrm{~m}$ above the bottom of the wheels when the rover is on a flat surface. The Mastcams (we use the plural here because the "eyes" of the fixed focal length (FFL) Mastcam investigation are not identical) consist of two cameras (Fig. 26) with different focal lengths and different science color filters. The stereo baseline of the pair is $\sim 24.5 \mathrm{~cm}$. One camera, referred to as the Mastcam-34 (M-34), has a $\sim 34 \mathrm{~mm}$ focal length, $\mathrm{f} / 8$ lens that observes a $15^{\circ}$ square field-of-view (FOV), to create a pixel scale of $450 \mu \mathrm{m}$ at $2 \mathrm{~m}$ distance and $22 \mathrm{~cm}$ at $1 \mathrm{~km}$. The other camera, the Mastcam100 (M-100), has a $\sim 100 \mathrm{~mm}$ focal length, f/10 lens with a $5.1^{\circ}$ square FOV with a pixel scale of $7.4 \mathrm{~cm}$ at $1 \mathrm{~km}$ distance and $\sim 150 \mu \mathrm{m} /$ pixel scale at $2 \mathrm{~m}$ distance. Both cameras can focus from the nearest view to the surface to infinity. The M-34 can focus as close as $0.34 \mathrm{~m}$, but the M-100 can only focus as close as $1.63 \mathrm{~m}$.

Each camera is capable of acquiring images at very high frame rates compared to previous missions, including 720 p high definition video (1280 by 720 pixels) at $\sim 8$ frames per second, and full science frames at somewhat less than $4 \mathrm{fps}$. Radiometric accuracy is $\leq 10-15 \%$, and precision $5-8 \%$.

The primary objectives of the Mastcam investigation are to characterize and determine details of the history and processes recorded by the geology the Gale crater field site. This includes but is not limited to observations of landscape that enable understanding of past and present geologic processes; studies of frost, ice, and related processes; documentation of atmospheric and meteorological events; and helping to facilitate rover operations, analytical laboratory sampling, contact instrument science.

See Malin et al. (in preparation) for additional description of the Mastcam instruments. 

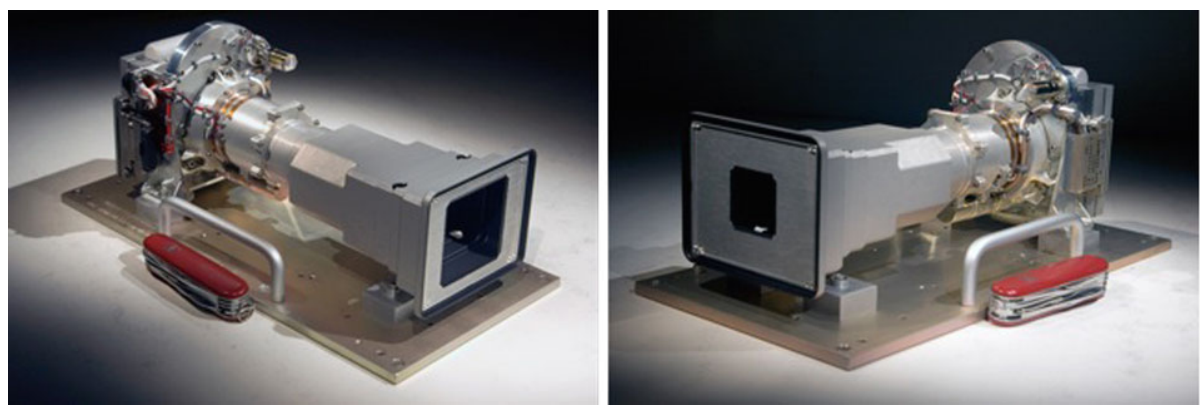

Fig. 26 Flight models of fixed-focal length (FFL) Mastcams. The only distinguishing difference in outward appearance between the cameras is the aperture size in the front baffle, which relates to the focal length of the lens. Mastcam-34 (on left) has a $\sim 34 \mathrm{~mm}$ focal length lens, and the Mastcam-100 (on right) has a $\sim 100 \mathrm{~mm}$ focal length lens

\subsection{RAD (Radiation Assessment Detector)}

The Radiation Assessment Detector (RAD) is an energetic particle analyzer designed to characterize the full spectrum of energetic particle radiation at the surface of Mars, including galactic cosmic rays (GCRs), solar energetic particles (SEPs), secondary neutrons and other particles created both in the atmosphere and in the Martian regolith. RAD's primary science objectives are to characterize the energetic particle spectrum at the surface of Mars; determine the radiation dose rate for humans on the surface of Mars; enable validation of Mars atmospheric transmission models and radiation transport codes; provide input to the determination of the radiation hazard and mutagenic influences to life, past and present, at and beneath the Martian surface, and provide input to the determination of the chemical and isotopic effects of energetic particles on the Martian surface and atmosphere.

The RAD instrument (Fig. 27) consists of a charged particle telescope comprised of three solid-state detectors and a cesium iodide (CsI) calorimeter. An additional BC-432 scintillating plastic channel is used together with the CsI calorimeter and an anti-anticoincidence shield to detect and characterize neutral particles (i.e., neutrons and gamma rays). The outputs of the various photodiodes, used with the CsI and scintillating plastic, and solid-state detectors are converted to digital pulse height discriminated signals for further processing. The RAD instrument is mounted just below the top deck of the rover with the charged particle telescope pointed in the zenith direction.

The RAD instrument began nominal operations during cruise on December 6, and will be used throughout the mission, to characterize the radiation environment of MSL. Due to rover energy constraints, RAD will acquire roughly 15 -minute observations every hour throughout each sol during surface operations.

The RAD instrument is discussed in detail in Hassler et al. (2012, this issue).

\subsection{REMS (Rover Environmental Monitoring Station)}

The main science objectives of the REMS investigation are to assess the general circulation and mesoscale phenomena near the surface of Mars (e.g., fronts, jets); identify microscale weather systems (e.g., boundary layer turbulence, heat fluxes, dust devils); describe the local hydrological cycle (e.g., spatial and temporal variability, diffusive transport from regolith); measure the destructive potential of UV radiation, dust UV optical properties, photolysis 
Fig. 27 RAD flight model in the lab. The RAD charged particle channel has a $65^{\circ}$ field-of-view pointing toward the zenith

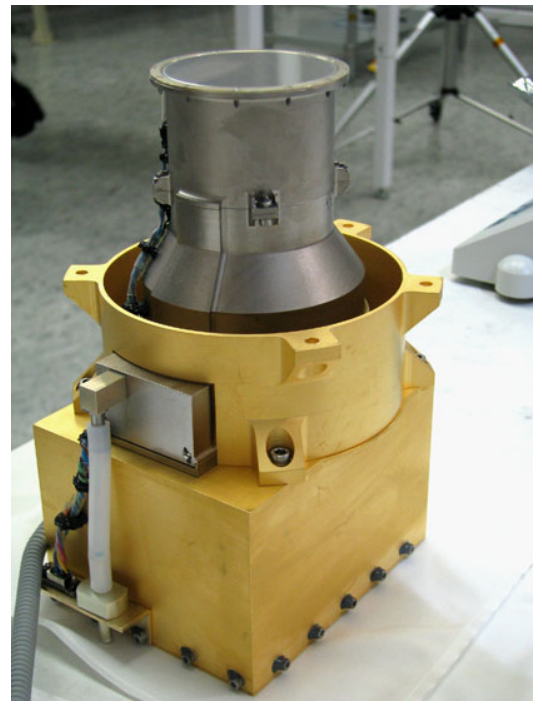

rates, and oxidant production; assess subsurface habitability based on ground-atmosphere interaction.

REMS has been designed to record six atmospheric parameters: wind speed/direction, pressure, relative humidity, air temperature, ground temperature, and ultraviolet radiation. All sensors are located around three elements: two booms attached to the rover Remote Sensing Mast, the Ultraviolet Sensor assembly located on the rover top deck, and the Instrument Control Unit inside the rover body. The booms are approximately $1.5 \mathrm{~m}$ above ground level and are separated in azimuth by $120^{\circ}$ to help insure that at least one of them will record clean wind data for any given wind direction. Figure 28 shows the booms' relative position. There is a $50 \mathrm{~mm}$ height difference to minimize mutual wind perturbation. Boom 2, which points in the driving direction of the rover, has wind sensors and the relative humidity sensor. Boom 1, which looks to the side and slightly to the rear of the rover, hosts another set of wind sensors and the ground temperature sensor. Both booms have an air temperature sensor.

Wind speed and direction will be derived based on information provided by three twodimensional wind sensors on each of the booms. Each of them will record local speed and direction in the plane of the sensor, with an accuracy of $1 \mathrm{~m} / \mathrm{s}$ and 30 degrees respectively; a specific chamber has been developed for calibration, reproducing atmosphere pressure and composition. Ground temperature will be recorded with a thermopile that views the Martian surface to the side of the rover (Sebastián et al. 2010), the accuracy expected for those data is better than $10 \mathrm{~K}$; a set-up based on a cryostat and a black body has been used for its calibration. Air temperature will be recorded by a sensor placed on a small rod long enough to be outside the mast and boom thermal boundary layers, with an accuracy of $5 \mathrm{~K}$.

Boom 2 houses the humidity sensor, which is located inside a protective cylinder. That sensor will measure relative humidity with an accuracy of $10 \%$ in the $200-323 \mathrm{~K}$ range and with a resolution of $1 \%$. A dust filter protects it from dust deposition. The UV sensor will be located on the rover deck and is composed of six photodiodes that face the zenith direction and have a field of view of $60^{\circ}$. The pressure sensor will be located inside the rover body and connected to the external atmosphere via a tube. 


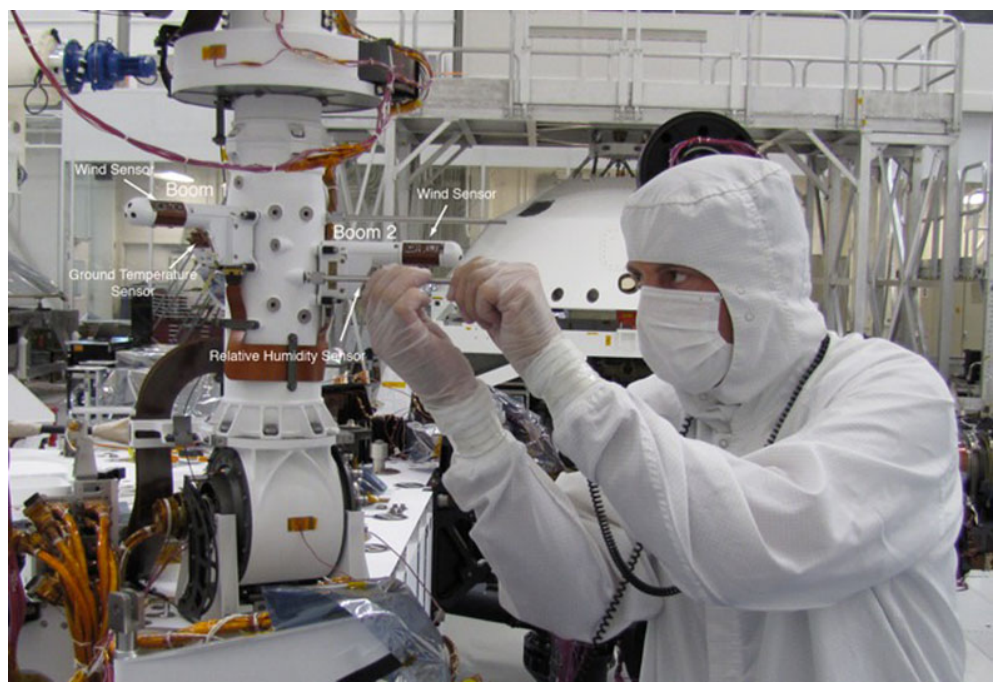

Fig. 28 REMS flight booms installed on a section of the rover mast. On the left is Boom 1 with wind and ground temperature sensor and on the right is Boom 2 with wind and humidity sensors. The humidity sensor itself extends downward from the boom and is hidden by the engineer's right hand. On both booms the conditioning electronics are located on the back, close to the attachment point on the mast

Systematic measurement is the main driver for REMS operation. Data will be recorded each hour, every sol, for 5 minutes at $1 \mathrm{~Hz}$ for all sensors. The instrument will wake itself up each hour and after recording and storing data, will go to sleep independently of rover operations. REMS will record data whether the rover is awake or not, and both day and night.

The REMS instrument is discussed in detail in Gomez-Elvira (2012, this issue).

\subsection{SAM (Sample Analysis at Mars Instrument Suite)}

The Sample Analysis at Mars (SAM) Suite Investigation in the MSL Analytical Laboratory is designed to address the present and past habitability of Mars by exploring molecular and elemental chemistry relevant to life. SAM addresses carbon chemistry through a search for organic compounds, the chemical state of light elements other than carbon, and isotopic tracers of planetary change.

SAM is a suite of three instruments (see Fig. 29), a Quadrupole Mass Spectrometer (QMS), a Gas Chromatograph (GC), and a Tunable Laser Spectrometer (TLS). The QMS and the GC can operate together in a GCMS mode for separation (GC) and definitive identification (QMS) of organic compounds. The QMS is the primary detector for the GC with a mass range of 2-535 Dalton, and can analyze the atmosphere or gases thermally evolved from solid phase samples (rock powder or soil). The TLS obtains precise isotope ratios for C and $\mathrm{O}$ in carbon dioxide and measures trace levels of methane- to 1-5 ppb levels-and its carbon isotope. The GC separates complex mixtures of organic compounds into molecular components for QMS and GC stand-alone analysis; helium is the carrier gas.

The three SAM instruments are supported by a sample manipulation system (SMS) and a Chemical Separation and Processing Laboratory (CSPL) that includes high conductance and micro valves, gas manifolds with heaters and temperature monitors, chemical and mechanical pumps, carrier gas reservoirs and regulators, pressure monitors, pyrolysis ovens, 
Fig. 29 Flight model of SAM instrument. QMS, quadrapole mass spectrometer; TLS, tunable laser spectrometer; GC, gas chromatograph (all six are visible). The instrument is $\sim 55 \mathrm{~cm}$ wide and $\sim 31 \mathrm{~cm}$ tall

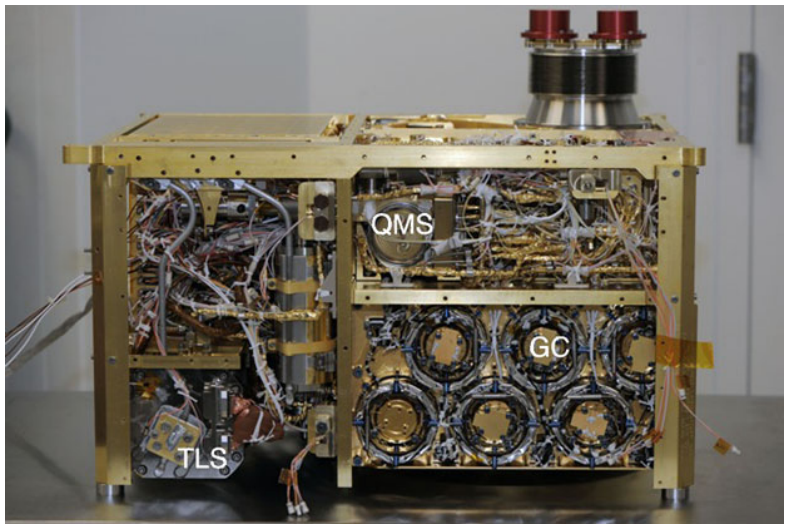

and chemical scrubbers and getters. The Mars atmosphere is sampled by CSPL valve and pump manipulations that introduce an appropriate amount of gas through an inlet tube to the SAM instruments. The solid phase materials are sampled by transporting finely sieved materials to one of 74 SMS sample cups that can then be inserted into a SAM oven and thermally processed for release of volatiles. The volume of sample that can be accommodated by the SAM quartz cups is more than 6 times the maximum volume delivered in a single sample drop from the SA/SPaH portioner. Thus, multiple samples could be delivered to the SAM quartz cups prior to an evolved gas experiment or subsequent experiments could deposit fresh sample onto the de-volatilized residue from a previous sample run.

The SAM instrument is discussed in detail in Mahaffy et al. (2012, this issue).

\section{Mission Planning}

The following sections describe the phases of the MSL mission, from launch through the end of surface operations. The timeline and primary activities are summarized for each phase. For the surface phase, these are accompanied by an overview of the various constraints on science operations and a description of mission scenarios that exemplify how science return can be optimized under them.

\subsection{Launch, Cruise, Approach, and EDL Phases}

The Atlas V (541) launch vehicle and Centaur upper stage injected the spacecraft on a Type I trajectory (see Fig. 30) for arrival at Mars on 6 August 2012 (05:05 to 05:18 UTC). During the cruise to Mars, the spacecraft performed three trajectory correction maneuvers before July 15, 2012, to correct for launch vehicle injection errors, remove the initial bias required for planetary protection, and refine the trajectory toward the entry aim point at Mars (see Fig. 31). During this time a number of maintenance and checkout activities were performed. A health checkout of each of the science instruments plus the engineering cameras was carried out during the period of March 12-22, 2012, beginning 108 days after launch. The one exception to this is RAD, which was checked out and began routine science observations on December 6, 10 days after launch. Additional late cruise checkouts were performed on Mastcan, MARDI, MAHLI and the engineering cameras on April 20, 2012; Mastcam, MARDI and MAHLI plus the engineering cameras and REMS were checked again on June 


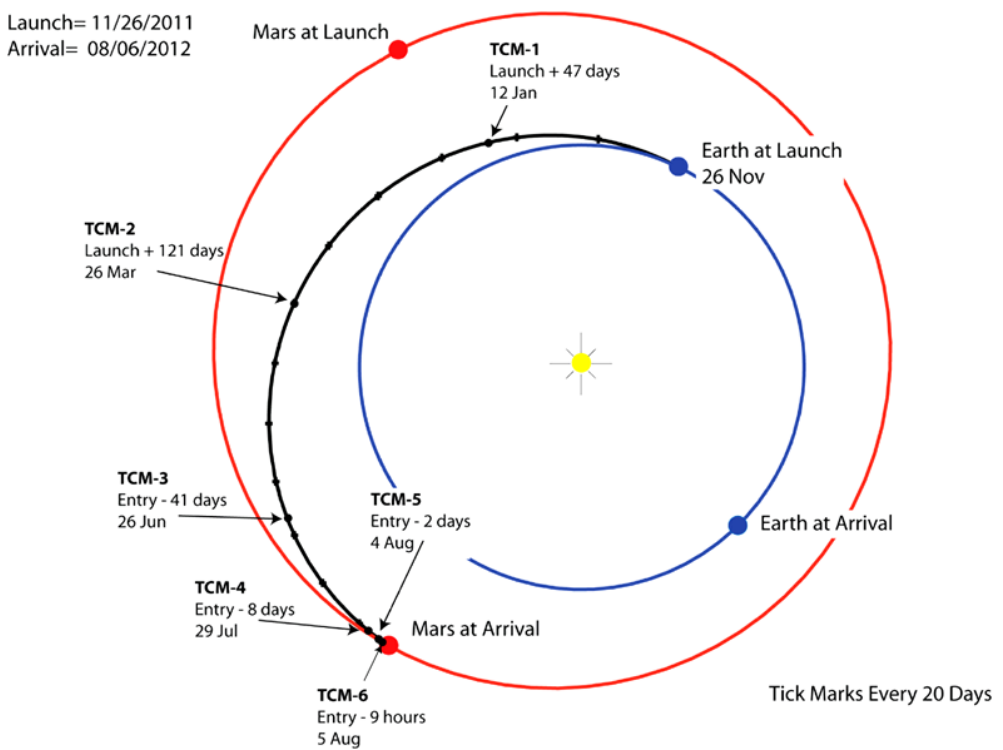

Fig. 30 MSL Interplanetary Trajectory: Type 1A/1B (Open of Launch Period). Note: A "Type 1" launch is defined by the spacecraft reaching Mars before it has traveled 180 degrees around Earth. TCM; Trajectory correction maneuver

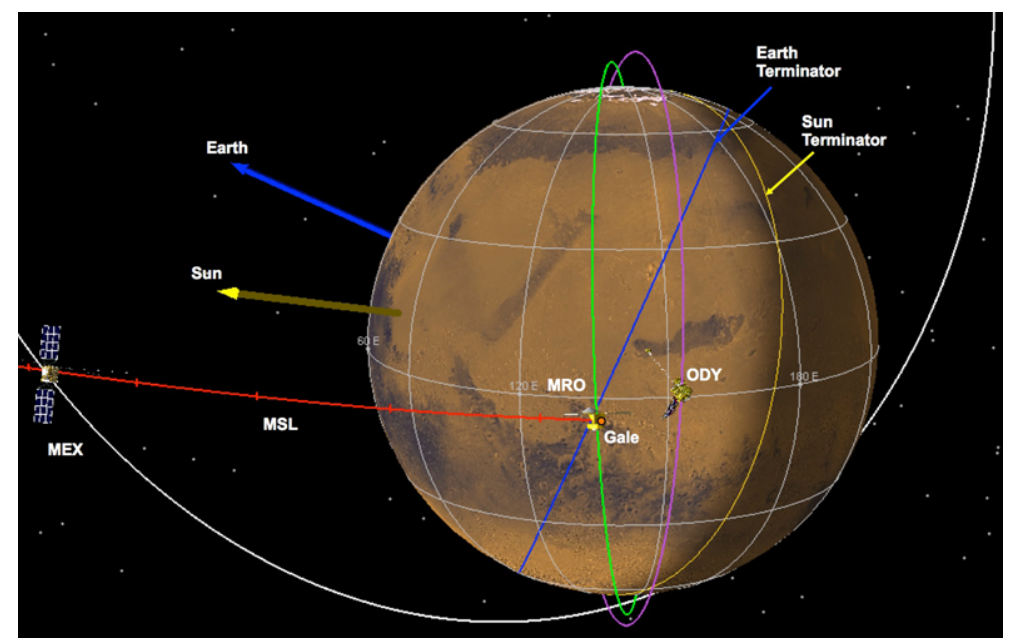

Fig. 31 View of EDL coverage geometry targeted to Gale crater, at time of spacecraft entry

14,2012 ; the SAM instrument was checked on June 28, 2012. The last 45 days before landing comprised the approach phase, involving additional trajectory correction maneuvers.

Entry, descent, and landing activities occur within $\sim 15$ minutes prior to touchdown on Mars. MARDI acquires its data set from moments before heat shield separation through touchdown $(<2$ minutes) and a few seconds thereafter. For landing, MSL uses a propulsive descent "sky crane" to lower the tethered rover beneath it onto the Martian surface, setting its wheels directly on the ground. After rover landing, the connection with the descent stage 
is severed and the descent stage flies away to fall elsewhere, $150 \mathrm{~m}$ or more away from the rover. The rover will touch down in late southern winter $\left(L_{s}=150.7\right)$, between 14:50 and 15:02 Local Mean Solar Time on Mars, depending on the launch date.

\subsection{Commissioning Phase}

The goal of the commissioning phase is to allow the mission to reach nominal science operations as quickly and safely as possible after touching down. Before this is possible, the rover operations team must characterize the health and behavior of the rover and instruments once interacting with the Martian environment. The first $\sim 10$ sols will be dominated by critical hardware deployments (e.g., the mast and mobility system), installing the flight software version used for surface operations, and spacecraft and payload checkout activities. After this initial characterization phase, mast-mounted and monitoring instruments may begin performing nominal science as operational time, power, and downlink data resources allow. The next few 10s of sols will involve checkouts and first-time uses of more advanced capabilities, such as the robotic arm and other sampling hardware. These activities will be coordinated with strategic science decisions such as whether to drive out of the region contaminated by the landing engines' effluents, to fully enable contact science, to acquire a sample of soil, rock, or organic check material, or to focus on traversing toward scientific targets.

\subsection{Surface Operations: Overview}

MSL's primary mission spans one Mars year (669 sols or 687 Earth days) after touchdown. Science team activities will terminate six months after the end of the surface mission, whether it ends after one Mars year or after any number of extensions. Nominal science operations will occur throughout this period with a few exceptions-namely, the commissioning phase right after landing, a 30-sol period of minimal operations centered on superior solar conjunction (18 April 2013), $\sim 10$ sols dedicated to software updates throughout the primary mission, and a few other maintenance activities.

MSL is intended to be a discovery-driven mission, with the science operations team retaining flexibility in how and when the various capabilities of the rover and payload are used to accomplish the overall scientific objectives. One major partition in the rover's activities is between driving and "sampling," where the latter represents a series of environmental, remote sensing, and contact science measurements may then lead to the acquisition, processing, and analysis of a sample of rock or soil in the analytical laboratories. The proximity of the specific touchdown location to targets of scientific interest within the landing ellipse, and to Mount Sharp itself, will influence the ratio of driving to sampling in the early mission.

Science activities on any particular sol are governed by a number of constraints that are measured or predicted for that sol, such as the Earth-Mars geometry and local time phasing, timing of telecom windows, downlink data volume capability, the time profile of energy available for science, and any thermally driven operational constraints or energy needs of the payload, rover subsystems necessary for payload operations (e.g., robotic arm actuators), or the rover. Science activities generally require more power than is available from the RTG and rely on drawing down the rover batteries. (This is also true for many engineering activities.) Battery capacity, RTG output, overnight battery recharge, and management of the state-ofcharge over multiple sols, are all critical to science (and engineering) planning. The thermal limitations, including significant time and energy required to heat mast, arm, and mobility actuators, vary with both time of day and season. 
Table 8 Tactical operations timelines for Mars-time and Earth-time mission schedules


Most science activities will occur during daylight hours on Mars (Table 8). Throughout the mission the rover itself operates on Mars time. Commands for the sol's activities are sent via the overnight orbiter telecom pass or direct-from-Earth at local midmorning on Mars. The rover will complete its tactical science activities (i.e., those that influence planning for the next sol) in time to return the data via an orbiter telecom pass in the midafternoon. During the early portion of the mission, the operations team will synchronize its efforts to Mars time. Between midafternoon and the next morning on Mars, the science operations team on Earth will assess the downlink, plan the next sol's activities, and prepare the commands. Data that are not essential for next-sol planning will be returned during the overnight orbiter telecom pass. This basic framework allows approximately five hours for tactical science activities by the rover on Mars. Additional payload or rover operations can occur outside of this window if they are not critical to the next sol's planning. Following the first 90 sols the operations team will revert to an Earth-based time schedule (Table 8).

During winter, the time available each sol for science operations may be reduced because of the need to use a greater share of energy to heat the rover actuators. Also, the largest actuators may not warm sufficiently until after the afternoon orbiter telecom pass. For this reason, winter operations may use every-other-sol commanding for more than 12 out of every 36 sols.

\subsubsection{Mission Operations After Landing}

The first $\sim 90$ days of the mission is accomplished with all operations participants on site at JPL, with personnel working in shifts synchronized to Mars' 24.6-h day and on duty around the clock, seven days a week. A description of how Mars Time operations worked for the Mars Exploration Rover mission is given in Bass et al. (2005) and Mishkin et al. (2006). Operating on Mars Time and extra staffing to cover the tactical uplink process will allow the extension of the tactical timeline from the normal 8 hours to a two-shift, 12 to 16 hour timeline (Table 8). A major objective of this period is to develop the capability to complete the tactical one-sol turnaround process in 8 hours or less, by increasing efficiency.

After a portion (or all) of this initial period, the flight team begins transitioning to operate via a distributed operations network, with the central hub at JPL. This enables the remote science teams to work from their home institutions for the long duration of the mission, interacting via internet and phone teleconferencing. The start time of the prime shift on Earth will track Mars time, sliding forward from 6 AM until it reaches 1 PM (Pacific). After 
Table 9 A few of the resources that drive science operations and the required minimum capabilities built into the design of MSL

Some resources that drive science operations

Required capability

Energy available for science activities

$250 \mathrm{~W} \mathrm{~h} / \mathrm{sol}$

Downlink volume (two UHF passes)

250 megabits/sol

Rover awake time

$6 \mathrm{~h} / \mathrm{sol}$

Traverse distance

$50 \mathrm{~m} / \mathrm{sol}$

Note: The actual capability on Mars may exceed the requirements for certain environmental conditions or other favorable conditions. The requirements may not be met during anomalous conditions. Science activities include rover traverses. A small fraction of the downlink volume will be used to transmit rover health and housekeeping data

this point, the downlink from Mars arrives too late in the day on Earth to allow commands to be generated before a reasonable end of shift. In these cases the ground cycle is postponed until the next available Earth shift. From a tactical standpoint, every other sol is lost during this period. However, science activities can be performed by the rover on every sol as long as they can be planned in advance and/or their results are not required immediately for future planning. This period of every-other-sol (or multiple-sol) commanding is expected to span about 12 sols of every 36-sol Earth-Mars phasing cycle. After the first six months of operations, the operations team will support 5 day per week tactical operations on Earth time, with multiple-sol rover plans prepared for weekends and holidays.

Table 9 summarizes some of the key resources affecting operations and the required capability for each.

\subsection{Example Mission Scenario and Sol Types}

There exists an enormous variety of ways in which the mission may unfold, because of the unknown nature of the discoveries, the flexibility of the scientific payload, and the capabilities of the rover. However, in order to understand how science operations can be optimized given the constraints listed above, the mission planning team developed a set of example sol types and integrated them into a mission scenario. This scenario contains typical science activities that address the scientific goals of the mission, including driving and the use of all instruments. The purpose of the scenario study was to integrate the science strategies of the selected investigations and to demonstrate that a representative surface mission fits within the mission constraints, not to examine every conceivable use of the payload or rover. The scenario study was performed early in the development phase and before landing site selection. At that time, it validated that the mission operations teams and infrastructure were capable of meeting mission performance requirements of at least 28 samples and $4.5 \mathrm{~km}$ of traverse. This analysis is now superseded by the performance study described in Sect. 5.5.

The mission scenario envisions a logical sequence of scientific operations that repeats multiple times as the rover explores the region within its field site. The rover performs a detailed examination of a number of distinct locations. The analysis of each location is assumed to consist of a traverse to a site of interest, remote sensing measurements to identify a target, a short approach drive to place the target within the robotic arm workspace, contact analyses to triage the target and determine whether to sample it, a set of activities that acquire rock or soil samples, process them, and deliver them to the analytical laboratory instruments, and finally, the analysis by those instruments. Each subset of activities is grouped together 
Table 10 Instrument activities on MSL sol types. The shaded boxes mark the highest-priority activities. Environmental monitoring by DAN (passive mode), REMS, and RAD occurs throughout, as resources allow

\begin{tabular}{|c|c|c|c|c|c|c|c|}
\hline & DAN & Mastcam & ChemCam & MAHLI & APXS & CheMin & SAM \\
\hline Traverse & $\begin{array}{c}\text { survey } \\
\text { during drive }\end{array}$ & $\begin{array}{l}\text { corridor and } \\
\text { end-of-drive } \\
\text { panoramas }\end{array}$ & $\begin{array}{l}\text { pre- and } \\
\text { post-drive } \\
\text { survey }\end{array}$ & & & & \\
\hline Recon & & $\begin{array}{c}\text { panoramic } \\
\text { imaging }\end{array}$ & survey & single target & $\begin{array}{c}\text { long } \\
\text { integration }\end{array}$ & & \\
\hline Approach & $\begin{array}{c}\text { high- } \\
\text { precision } \\
\text { measurement }\end{array}$ & $\begin{array}{l}\text { workspace } \\
\text { imaging }\end{array}$ & $\begin{array}{l}\text { pre-drive } \\
\text { survey }\end{array}$ & single target & $\begin{array}{c}\text { short } \\
\text { integration }\end{array}$ & & \\
\hline Contact & & $\begin{array}{c}\text { pre-and } \\
\text { post-brush } \\
\text { imaging; } \\
\text { multispectral }\end{array}$ & $\begin{array}{l}\text { analysis of } \\
\text { contact } \\
\text { target }\end{array}$ & $\begin{array}{c}\text { pre- and } \\
\text { post-brush } \\
\text { imaging of } \\
\text { contact } \\
\text { target }\end{array}$ & $\begin{array}{c}\text { pre- and } \\
\text { post-brush } \\
\text { analysis of } \\
\text { contact } \\
\text { target }\end{array}$ & & \\
\hline $\begin{array}{l}\text { Dilution I } \\
\text { Sampling }\end{array}$ & & $\begin{array}{l}\text { support } \\
\text { imaging }\end{array}$ & & & & $\begin{array}{c}\text { sample } \\
\text { analysis } \\
\text { (begin) }\end{array}$ & \\
\hline $\begin{array}{l}\text { Analysis / } \\
\text { Obs Tray }\end{array}$ & & $\begin{array}{l}\text { support } \\
\text { imaging }\end{array}$ & & $\begin{array}{l}\text { sample } \\
\text { analysis }\end{array}$ & $\begin{array}{l}\text { sample } \\
\text { analysis }\end{array}$ & $\begin{array}{c}\text { sample } \\
\text { analysis } \\
\text { (end) }\end{array}$ & $\begin{array}{l}\text { sample } \\
\text { analysis }\end{array}$ \\
\hline
\end{tabular}

as a sol type (Table 10). In this scenario, each target is assumed to undergo the full set of activities, though in practice, each step is a decision point that can go forward or restart the process (e.g., if the contact analyses suggest the target is not worth sampling). Note: Environmental monitoring by DAN (passive mode), REMS, and RAD occurs throughout, as resources allow.

The sol types defined for the MSL mission are as follows:

Traverse Sols are sols in which roving is the dominant activity. The roving capability is assumed to be $50 \mathrm{~m} / \mathrm{sol}$, but will vary with terrain, thermal constraints, and available energy. Traverse Sols begin with a set of targeted ChemCam observations. The roving goal is determined from engineering camera data (from a previous sol) as well as HiRISE imagery. Mastcam panoramas and DAN measurements are taken at intervals along the traverse. At the end of the traverse, the rover acquires Mastcam and Navcam panoramas, Hazcam stereo pairs, and a set of untargeted ChemCam observations.

Reconnaissance Sols initiate the detailed study of a site by returning remotely sensed "survey" observations that allow the science team to plan the next steps. Reconnaissance Sols begin with a set of targeted ChemCam observations, followed by an arm deployment (requiring Hazcam imagery from a previous sol), and acquisition of MAHLI images. The remaining activities collect Mastcam and Navcam panoramas. The APXS remains deployed overnight for a long integration.

Approach Sols are used to place a target (e.g., part of a rock or a patch of soil) within the robotic arm's workspace and to prepare for workspace activities. The target is identified on a previous sol and can be reached in a single sol if it is less than $\sim 10 \mathrm{~m}$ distant. Approach Sols begin with targeted ChemCam observations, a short APXS integration, and MAHLI images before the approach. After roving, Navcam and Hazcam images, and Mastcam spectral data, are collected within the workspace. DAN acquires active measurements during the approach and at the new location.

Contact Sols conduct scientific observations of a target with the arm-mounted instruments. A specific target selected from the approach data is analyzed with MAHLI and APXS. The target is then brushed and the measurements are repeated, though with a longer APXS 
integration. ChemCam and Mastcam take spectral observations to provide context to the target, while Hazcam images document the activities.

Sampling and Analysis Sols contain a set of activities with the end goal of placing solid sample material within CheMin and SAM. While it may be deemed unnecessary at some point, the scenarios presently assume that cross contamination is reduced in the drill and sample processing hardware by acquiring a "cleaning" sample before the "science" sample. First, a sample is acquired by the drill from a spot near the science target and fed through the appropriate sieve. (Note: if the sample is anticipated to have some chance of behaving in an unusual way it might first be placed on the observation tray, or passed through the funnel tool mounted at the front of the rover.) Next, the primary sample is obtained, sieved, and delivered to CheMin and SAM. Remaining sample material, if available, is placed on the observation tray for analysis by MAHLI and APXS. Between observations, the tray is brushed by the DRT. Finally, CheMin and SAM complete their analyses. Unlike the other Sol Types that have activities thought to fit within a single tactical window on Mars, these activities will span 3 to 5 sols because of the required time, energy, and data volume. As a rule of thumb, sample acquisition, CheMin analyses, and SAM analyses each require at least one sol's worth of resources.

In addition to the activities in the above sol types oriented toward selecting and analyzing solid samples, there are many additional activities critical to achieving the mission's science objectives. Examples include the analysis of atmospheric gases by SAM, meteorological imaging, dedicated campaigns for REMS, RAD, and DAN, and calibration or cleaning activities for all instruments. These activities will be performed at the direction of the Science Operations Working Group, in some cases taking advantage of sols not available for tactical commanding because of Earth-Mars phasing.

\subsection{Estimated Mission Performance at Gale Crater}

The Project has developed quantitative models of the above sol types that incorporate operational constraints, the energy and data volume usages of the instruments and rover, and the time required to perform the activities. Cases were run for three thermal environments corresponding to Winter, Spring/Fall, and Summer at a notional landing site at $27^{\circ} \mathrm{S}$. This latitude is near the extreme accessible to MSL, and being in the southern hemisphere, has a large seasonal variation driven by Mars' axial tilt and eccentricity. The Gale landing site has conditions all year round that are best approximated by the Spring/Fall case at $27{ }^{\circ} \mathrm{S}$, and those conditions are used for the present performance analysis. The thermal environment at Gale is further described by Vasavada et al. (2012, this issue).

The two key measures of mission performance are the number of solid samples analyzed by the analytical laboratory instruments and the total distance traversed. A profile of performance in these two dimensions versus time is computed using the quantitative sol type models and the following assumptions: A commissioning phase of 90 sols produces insignificant traverse and no samples. Forty additional sols are not counted because they are used for maintenance activities or occur during solar conjunction. One in four sols (a $25 \%$ margin) is non-productive due to unforeseen shortfalls in mission resources, failure to achieve planned outcomes, or communication problems. One in five sols is not commandable on the tactical cycle because of Earth-Mars phasing, occurs during weekends after sol 180, and/or is dedicated toward science activities not involv- 
Fig. 32 Estimated number of samples and traverse distance vs. time at Gale crater
Estimated Mission Performance at Gale

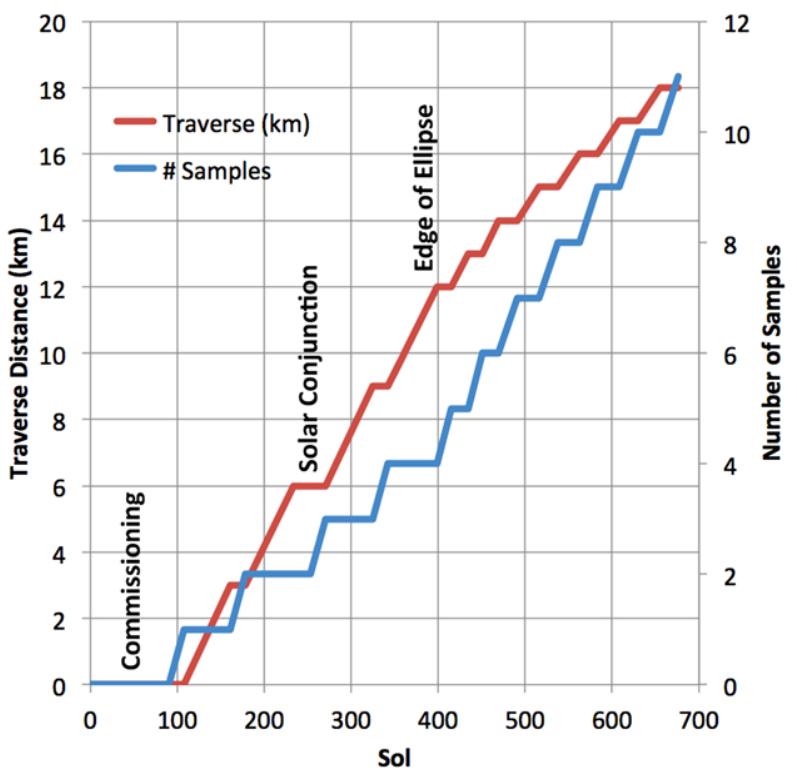

ing the solid-sample-oriented sol types. The remaining sols are used to perform the soltype scenario at Gale crater. Four samples are acquired within the ellipse. Afterward, one sample is acquired every kilometer. An average traverse rate of $50 \mathrm{~m} / \mathrm{sol}$ is assumed.

Given these assumptions, over its one Mars year primary mission, MSL is capable of selecting and analyzing 11 solid samples with a total traverse distance of $18 \mathrm{~km}$. Figure 32 shows how the progress in sampling and traversing occurs over time and in relation to the edge of the landing ellipse (coincident with the base of Mount Sharp). The curves can be used to judge tradeoffs between sampling and traversing, for example, another scenario might involve traversing as rapidly as possible to Mount Sharp, resulting in fewer early samples but more sols to explore and sample Mount Sharp. A separate study of traverse performance at Gale, taking into account the details of the terrain, is described in Sect. 2.6 of this paper.

\section{Planetary Protection}

In the study of whether Mars has had environments conducive to life, precautions must be taken to avoid introduction of microbes from Earth by robotic spacecraft. Consistent with this, the United States is a signatory to an international treaty stipulating that exploration must be conducted in a manner that avoids harmful contamination of celestial bodies. NASA has a Planetary Protection Office responsible for establishing and enforcing planetary protection regulations created to avoid biological contamination in the process of exploration. Each spacecraft mission is responsible for implementing measures to comply with the regulations. In compliance with the treaty and NASA regulations, the Mars Science Laboratory flight hardware has been designed and built to meet planetary protection requirements. 
NASA's primary strategy for preventing contamination of Mars with Earth organisms is to be sure that all hardware going to the planet is biologically clean. The Mars Science Laboratory mission is allowed to carry up to 500,000 bacterial spores on the entire flight system. Spore-forming bacteria have been the focus of planetary protection standards because these bacteria can survive harsh conditions for many years as inactive spores. One requirement for this mission is that the exposed interior and exterior surfaces of the landed system, which includes the rover, parachute and back shell, must not carry a total number of bacterial spores greater than 300,000 , with the average spore density not exceeding 300 spores per square meter. This ensures that biological load is not concentrated in one place. The heat shield and descent stage will hit the ground hard enough that hardware could break open. No more than 200,000 spores may be present that could be exposed by the hard landings of these components.

The standard of cleanliness is even stricter for portions of the rover's sample-acquisition hardware that will contact the Martian subsurface or the interior of rocks. While these components are baked to sterilize their surfaces using the same techniques as other hardware, special care is taken to prevent possible recontamination that can occur even in a cleanroom. Covering the hardware and use of special HEPA filter walls around the sampling hardware ensures this higher level of biological cleanliness.

The two primary methods used for reducing the number of spores on the spacecraft are alcohol wipe cleaning and dry heat microbial reduction. Technicians and engineers assembling the spacecraft and preparing it for launch have routinely cleaned surfaces by wiping them with alcohol and other solvents. Components tolerant of high temperature were heated to reduce spore burden according to NASA specification, at temperatures ranging from 110 to 146 degrees Celsius for durations up to 144 hours. The planetary protection team carefully sampled the surfaces and performed microbiological tests to demonstrate that the spacecraft met requirements for biological cleanliness.

The Mars Science Laboratory is also complying with a requirement to avoid going to any site on Mars known to have water or water-ice within one meter of the surface. This is a precaution against any landing-day accident that could introduce hardware not fully sterilized by dry heat into an environment where heat from the mission's radioisotope thermoelectric generator and a Martian water source could provide conditions favorable for microbes from Earth to grow on Mars.

Another way of making sure the mission does not transport Earth life to Mars is to ensure that any hardware not meeting cleanliness standards does not go to Mars accidentally. When the Atlas launch vehicle's upper stage Centaur separates from the spacecraft, the two objects are traveling on nearly identical trajectories. To prevent the possibility of the Centaur hitting Mars, that shared flight path is deliberately set so that the spacecraft would miss Mars if not for later maneuvers to adjust its trajectory. By design, the Centaur is never aimed at Mars.

Portions of the flight hardware will impact the surface of Mars as part of a normal landing event. This impact may cause the hardware to split open and potentially release spores trapped inside the hardware during manufacturing processes. To ensure MSL does not exceed the spore allocation, studies were conducted on various materials, including paint, propellants and adhesives, to determine the number of spores in a given volume. In many cases the parts of the spacecraft containing these materials used dry heat microbial reduction to reduce the number of spores. For hardware expected to impact Mars, such as the cruise stage after its separation from the aeroshell, a detailed thermal analysis was conducted to make sure that plunging through Mars atmosphere gets it sufficiently hot that few to no spores survive. 


\section{Summary}

The Mars Science Laboratory Mission represents an ambitious step forward in the exploration of Mars. The synergistic interplay of sequential and overlapping orbiter and rover missions has dramatically improved our understanding of the history of Mars surface environments, including those which may have been habitable by microorganisms, had life evolved on Mars. The Curiosity rover now will explore one of the most intriguing of those possibly habitable environments at Gale crater, documenting not only the capability of past and present environments to support life, but also chronicling the history of Mars' ancient environments including major changes that transformed the planet from wetter, more clement conditions into its currently dry state. A stack of sedimentary strata that form Mount Sharp, exceeding 5 kilometers in thickness, will be interrogated by Curiosity's payload to provide a relative time series of geochemical and geological variability that will hopefully contain important clues regarding the evolution of former surface environments. Simultaneously, the modern environment will be monitored in order to better understand daily and seasonal variability, the nature of atmospheric processes, and the flux of background solar and cosmic radiation. Additional information can be found at the MSL website (http://mars.jpl.nasa.gov/msl).

Acknowledgements We acknowledge the contributions of previous MSL Project Scientists, Frank Palluconi and Edward Stolper, previous REMS PI Luis Vazquez, and the hundreds of scientists and engineers around the world that have been involved in the development of MSL and its scientific payload. Paulo Bellutta carried out the traversability studies. Fernando Abilleira contributed mission design and navigation diagrams. Part of this research was carried out at the Jet Propulsion Laboratory, California Institute of Technology, under a contract with the National Aeronautics and Space Administration.

Open Access This article is distributed under the terms of the Creative Commons Attribution License which permits any use, distribution, and reproduction in any medium, provided the original author(s) and the source are credited.

\section{References}

F. Abilleira, Exploration and utilization of extraterrestrial bodies, in Proceedings of the 12th International Conference on Engineering, Science, Construction, and Operations in Challenging Environments. Earth and Space 2010 (American Society of Civil Engineers, Reston, 2010). Symposium 4. doi:10.1061/41096(366)85

R.B. Anderson, J.F. Bell III, Geologic mapping and characterization of Gale Crater and implications for its potential as a Mars Science Laboratory landing site. Mars 5, 76-128 (2010). doi:10.1555/mars.2010.0004

R.C. Anderson, L. Jandura, A.B. Okon, D. Sunshine, C. Roumeliotis, L.W. Beegle, J. Hurowitz, B. Kennedy, D. Limonadi, S. McClowskey, M. Robinson, C. Seybold, K. Brown, Collecting samples in Gale Crater, Mars; an overview of the Mar Science Laboratory Sample Acquisition, Sample Processing and Handling system. Space Sci. Rev. (2012). doi:10.1007/s11214-012-9898-9, this issue

S.K. Atreya, P.R. Mahaffy, A.-S. Wong, Methane and related trace species on Mars: origin, loss, implications for life, and habitability. Planet. Space Sci. 55(3), 358-369 (2007)

D.S. Bass, R.C. Wales, V.L. Shalin, Choosing Mars time: analysis of the Mars Exploration Rover experience, in IEEE Aerospace Conference, 5-12 March (2005), pp. 4174-4185, paper \#1162. doi:10.1109/ AERO.2005.1559722

D. Blake, D. Vaniman, C. Achilles, R. Anderson, D. Bish, T. Bristow, C. Chen, S. Chipera, J. Crisp, D. Des Marais, R.T. Downs, J. Farmer, S. Feldman, M. Fonda, M. Gailhanou, H. Ma, D.W. Ming, R.V. Morris, P. Sarrazin, E. Stolper, A. Treimann, A. Yen, Characterization and calibration of the CheMin mineralogical instrument on Mars Science Laboratory. Space Sci. Rev. (2012). doi:10.1007/s11214-012-9905-1, this issue

J.L. Campbell, G.M. Perrett, R. Gellert, S.M. Andrushenko, N.I. Boyd, J.A. Maxwell, P.L. King, C.D.M. Schofield, Calibration of the Mars Science Laboratory Alpha Particle X-ray Spectrometer. Space Sci. Rev. (2012). doi:10.1007/s11214-0129873-5, this issue 
P.C. Conrad, J.L. Eigenbrode, M.O. Von der Heydt, C.T. Mogensen, J. Canham, D.H. Harpold, J. Johnson, T. Errigo, D.P. Glavin, P.R. Mahaffy, The Mars Science Laboratory organic check material. Space Sci. Rev. (2012). doi:10.1007/s11214-012-9893-1, this issue

D.J. Des Marais, Isotopic evolution of the biogeochemical carbon cycle during the Precambrian. Rev. Mineral. Geochem. 43(1), 555-578 (2001). doi:10.2138/gsrmg.43.1.555

K.S. Edgett, M.C. Malin, Martian sedimentary rock stratigraphy: outcrops and interbedded craters of northwest Sinus Meridiani and southwest Arabia Terra. Geophys. Res. Lett. 29 (2002)

K.S. Edgett, R.A Yingst, M.A. Ravine, M.A. Caplinger, J.N. Maki, F.T. Ghaemi, J.A. Schaffner (MAHLI team), Curiosity's Mars Hand Lens Imager (MAHLI) Investigation. Space Sci. Rev. (2012). doi:10.1007/s11214-012-9910-4, this issue

B.L. Ehlmann, J.F. Mustard, C.I. Fassett, S.C. Schon, J.W. Head III, D.J. Des Marais, J.A. Grant, S.L. Murchie, Clay minerals in delta deposits and organic preservation potential on Mars. Nat. Geosci. 1, 355-358 (2008)

C. Fabré, S. Maurice, V. Sautter, R. Wiens, J. Dubessy, M.C. Boiron, the ChemCam Team, Onboard calibration silicon targets for the ChemCam LIBS instrument (MSL rover), in Lunar Planetary Science Conf., vol. XL, abstract \#1502 (2009)

C. Fabré, S. Maurice, R. Wiens, V. Sautter, the ChemCam Team, ChemCam LIBS instrument: complete characterization of the onboard calibration silicate targets (MSL rover), in Lunar Planetary Science Conf., vol. XLI, abstract \#1835 (2010)

R.L. Fergason, P.R. Christensen, M.P. Golombek, T.J. Parker, Surface properties of the Mars Science Laboratory candidate landing sites: characterization from orbit and predictions. Space Sci. Rev. (2012). doi:10.1007/s11214-012-9891-3, this issue

R. Gellert, R. Rieder, J. Brückner, B.C. Clark, G., Dreibus, G., Klingelhöfer, G., Lugmair, D.W., Ming, H., Wänke, A., Yen, J., Zipfel, S.W., Squyres, Alpha particle X-ray spectrometer (APXS): results from Gusev Crater and calibration report. J. Geophys. Res. 111, E02S05 (2006). doi:10.1029/2005JE002555

T.D. Glotch, J.L. Bandfield, P.R. Christensen, W.M. Calvin, S.M. McClennan, B.C. Clark, D. Rogers, S. Squyres, Mineralogy of the light-toned outcrop at Meridiani Planum as seen by the Miniature Thermal Emission Spectrometer and implications for its formation. J. Geophys. Res. (2006). doi:10.1029/2005JE002672

M. Golombek, J. Grant, D. Kipp, A. Vasavada, R. Kirk, R. Fergason, P. Bellutta, F. Calef, K. Larsen, Y. Katayama, A. Huertas, R. Beyer, A. Chen, T. Parker, B. Pollard, S. Lee, Y. Sun, R. Hoover, H. Sladek, J. Grotzinger, R. Welch, E. Noe Dobrea, J. Michalski, M. Watkins, Selection of the Mars Science Laboratory Landing Site. Space Sci. Rev. (2012). doi:10.1007/s11214-012-9916-y, this issue

J. Gomez-Elvira (REMS team), REMS: an environmental sensor suite for the Mars Science Laboratory rover. Space Sci. Rev. (2012), this issue

J.P. Grotzinger, R.E. Milliken, The sedimentary rock record of Mars: distribution, origins, and global stratigraphy, in Sedimentary Geology of Mars, ed. by J.P. Grotzinger, R.E. Milliken, SEPM Special Paper (2012)

R.M. Hazen, D. Papineau, W. Bleeker, R.T. Downs, J.M. Ferry, T.J. McCoy, D.A. Sverjensky, H. Yang, Mineral evolution. Am. Mineral. 93(11-12), 1693-1720 (2008). doi:10.2138/am.2008.2955

D.M. Hassler, C. Zeitlin, R.F. Wimmer-Schweingruber, S. Böttcher, C. Martin, J. Andrews, E. Böhm, D.E. Brinza, M.A. Bullock, S. Burmeister, B. Ehresmann, M. Epperly, D. Grinspoon, J. Köhler, O. Kortmann, K. Neal, J. Peterson, A. Posner, S. Rafkin, L. Seimetz, K.D. Smith, Y. Tyler, G. Weigle, G. Reitz, F.A. Cucinotta, The Radiation Assessment Detector (RAD) investigation. Space Sci. Rev. (2012). doi:10.1007/s11214-012-9913-1, this issue

S.W. Hobbs, D.J. Paull, M.C. Bourke, Aeolian processes and dune morphology in Gale Crater. Icarus 210, 102-115 (2010). doi:10.1016/j.icarus.2010.06.006

D.M. Hunten, Possible oxidant sources in the atmosphere and surface of Mars. J. Mol. Evol. 14(1-3), 71-78 (1979). doi:10.1007/BF01732369

A.H. Knoll, The geological consequences of evolution. Geobiology 1(1), 3-14 (2003)

M.L. Litvak, I.G. Mitrofanov, Y.N. Barmakov, A. Behar, A. Bitulev, Y. Bobrovnitsky, E.P. Bogolubov, W.V. Boynton, S.I. Bragin, S. Churin, A.S. Grebennikov, A. Konovalov, A.S. Kozyrev, I.G. Kurdumov, A. Krylov, Y.P. Kuznetsov, A.V. Malakhov, M.I. Mokrousov, V.I. Ryzhkov, A.B. Sanin, V.N. Shvetsov, G.A. Smirnov, S. Sholeninov, G.N. Timoshenko, T.M. Tomilina, D.V. Tuvakin, V.I. Tretyakov, V.S. Troshin, V.N. Uvarov, A. Varenikov, A. Vostrukhin, The Dynamic Albedo of Neutrons (DAN) experiment for NASA's 2009 Mars Science Laboratory. Astrobiology 8(3), 605-612 (2008). doi:10.1089/ast.2007.0157

P.M. Mahaffy, C.R. Webster, M. Cabane, P.C. Conrad, P. Coll, SAM team, The Sample Analysis at Mars Investigation and instrument suite. Space Sci. Rev. (2012). doi:10.1007/s11214-012-9879-z, this issue 
J.N. Maki, J.F. Bell, K.E. Herkenhoff, S.W. Squyres, A. Kiely, M. Klimesh, M. Schwochert, T. Litwin, R. Willson, A. Johnson, M. Maimone, E. Baumgartner, A. Collins, M. Wadsworth, S.T. Elliot, A. Dingizian, D. Brown, E.C. Hagerott, L. Scherr, R. Deen, D. Alexander, J. Lorre, Mars Exploration Rover engineering cameras. J. Geophys. Res. Planets 108(E12), 8071 (2003). doi:10.1029/2003JE002077

J. Maki, D. Thiesses, A. Pourangi, P. Kobzeff, T. Litwin, L. Scherr, S. Elliott, A. Dingizian, M. Maimone, The Mars Science Laboratory engineering cameras. Space Sci. Rev. (2012). doi:10.1007/ s11214-012-9882-4, this issue

M.C. Malin, K.S. Edgett, Sedimentary Rocks of Early Mars. Science 290, 1927-1937 (2000)

M.C. Malin, K.S. Edgett, Evidence for persistent flow and aqueous sedimentation on Early Mars. Science 302, 1931-1934 (2003)

S. Maurice, R.C. Wiens, M. Saccoccio, B. Barraclough, O. Gasnault, O. Forni, N. Mangold, D. Baratoux, S. Bender, G. Berger, J. Bernardin, M. Berthé, N. Bridges, D. Blaney, M. Bouyé, P. Caïs, B. Clark, S. Clegg, A. Cousin, D. Cremers, A. Cros, L. DeFlores, C. Derycke, B. Dingler, G. Dromart, B. Dubois, M. Dupieux, E. Durand, L. d'Uston, C. Fabre, B. Faure, A. Gaboriaud, T. Gharsa, K. Herkenhoff, E. Kan, L. Kirkland, D. Kouach, J.-L. Lacour, Y. Langevin, J. Lasue, S. Le Mouélic, M. Lescure, E. Lewin, D. Limonadi, G. Manhès, P. Mauchien, C. McKay, P.-Y. Meslin, Y. Michel, E. Miller, H.E. Newsom, G. Orttner, A. Paillet, L. Parès, Y. Parot, R. Pérez, P. Pinet, F. Poitrasson, B. Quertier, B. Sallé, C. Sotin, V. Sautter, H. Séran, J.J. Simmonds, J.-B. Sirven, R. Stiglich, N. Striebig, J.-J. Thocaven, M.J. Toplis, D. Vaniman, The ChemCam instrument suite on the Mars Science Laboratory (MSL) rover: science objectives and mast unit description. Space Sci. Rev. (2012). doi:10.1007/s11214-012-9912-2, this issue

S. McLennan, J.P. Grotzinger, The sedimentary rock cycle of Mars, in The Martian Surface, ed. by J. Bell (Cambridge University Press, Cambridge, 2008), pp. 541-577

R.E. Milliken, J.P. Grotzinger, B.J. Thomson, Paleoclimate of Mars as captured by the stratigraphic record in Gale Crater. Geophys. Res. Lett. 37, L04201 (2010). doi:10.1029/2009GL041870

D.W. Ming, R. Gellert, R.V. Morris, R.E. Arvidson, J. Bruckner, B.C. Clark, B.A. Cohen, C. d'Uston, T. Economou, I. Fleischer, G. Klingelhofer, T.J. McCoy, D.W. Mittlefehldt, M.E. Schmidt, C. Schroder, S.W. Squyres, E. Treguier, A.S. Yen, J. Zipfel, Geochemical properties of rocks and soils in Gusev Crater, Mars: results of the Alpha particle X-ray spectrometer from Cumberland Ridge to Home Plate. J. Geophys. Res. Planets 113, E12S39 (2008). doi:10.1029/2008JE003195

A.H. Mishkin, D. Limonadi, S.L. Laubach, D.S. Bass, Working the Martian night shift-the MER surface operations process. IEEE Robot. Autom. Mag. 13(2), 46-53 (2006). doi:10.1109/MRA.2006.1638015

J.M. Moore, A.D. Howard, Large alluvial fans on Mars. J. Geophys. Res. 110, E04005 (2005). doi:10.1029/2004JE002352

M.J. Mumma, G.L. Villanueva, R.E. Novak, T. Hewagama, B.P. Bonev, M.A. SiSanti, A.M. Mandell, M.D. Smith, Strong release of methane on Mars in northern summer 2003. Science 323(5917), 1041-1045 (2009). doi:10.1126/science.1165243

J.F. Mustard, S.L. Murchie, S.M. Pelkey, B.L. Ehlmann, R.E. Milliken, J.A. Grant, J.-P. Bibring, F. Poulet, J. Bishop, E.N. Dobrea, L. Roach, F.P. Seelos, R.E. Arvidson, S. Wiseman, R. Green, C. Hash, D. Humm, E. Malaret, J.A. Mcgovern, K. Seelos, T. Clancy, R. Clark, D. Des Marais, N. Izenberg, S. Knudson, Y. Langevin, T. Martin, P. Mcguire, R. Morris, M. Robinson, T. Roush, M. Smith, G. Swayze, H. Taylor, T. Titus, M. Wolff, Hydrated silicate minerals on Mars observed by the Mars Reconnaissance Orbiter CRISM instrument. Nature 454, 305-309 (2008)

R. Navarro-Gonzalez, F.A. Rainey, P. Molina, D.R. Bagaley, B.J. Hollen, J. de la Rosa, A.M. Small, R.C. Quinn, F.J. Grunthaner, L. Cacares, B. Gomez-Silva, C.P. McKay, Mars-like soil in the Atacama Desert, Chile, and the dry limit of microbial life. Science 307(5647), 1018-1021 (2003). doi:10.1126/science. 1089143

F. Poulet, J.-P. Bibring, J.F. Mustard, A. Gendrin, N. Mangold, Y. Langevin, R.E. Arvidson, B. Gondet, C. Gomez, the OMEGA team, Phyllosilicates on Mars and implications for early Martian climate. Nature 438, 623-627 (2005)

R. Rieder, H. Waenke, T. Economou, A. Turkevich, Determination of the chemical composition of Martian soil and rocks: the alpha proton X-ray spectrometer. J. Geophys. Res. 102(E2), 4027-4044 (1997)

R. Rieder, R. Gellert, J. Brückner, G. Klingelhöfer, G. Dreibus, A. Yen, S.W. Squyres, The new Athena alpha particle X-ray spectrometer for the Mars Exploration Rovers. J. Geophys. Res. 108(E12), 8066 (2003). doi:10.1029/2003JE002150

E. Sebastián, C. Armiens, J. Gómez-Elvira, Pyrometer model based on sensor physical structure and thermal operation. Appl. Therm. Eng. 30, 2403-2411 (2010). doi:10.1016/j.applthermaleng.2010. 06.010

A.D. Steltzner, P.D. Burkhart, A. Chen, K.A. Comeaux, C.S. Guernsey, D.M. Kipp, L.V. Lorenzoni, G.F. Mendeck, R.W. Powell, T.P. Rivellini, A.M. San Martin, S.W. Sell, R. Prakash, D.W. Way, Mars science laboratory entry, descent, and landing system overview, in International Planetary Probe Workshop, Barcelona, Spain, June 12 (2010) 
R.E. Summons, J.P. Amend, D. Bish, R. Buick, G.D. Cody, D.J. Des Marais, G. Dromart, J.L. Eigenbrode, A.H. Knoll, D.Y. Sumner, Preservation of Martian organic and environmental records: final report of the Mars Biosignature Working Group. Astrobiology 11, 2 (2011). doi:10.1089/ast.2010.0506

D.Y. Sumner, Poor preservation potential of organics in Meridiani Planum hematite-bearing sedimentary rocks. J. Geophys. Res. 109, E12007 (2004). doi:10.1029/2004JE002321

I.L. ten Kate, J. Canham, P.G. Conrad, T. Errigo, I. Katz, P.R., Mitigation of the impact of terrestrial contamination on organic measurements from the Mars Science Laboratory. Astrobiology 8(3), 571-582 (2008). doi:10.1089/ast.2007.0160

B.J. Thomson, N.T. Bridges, R. Milliken, A. Baldridge, S.J. Hook, J.K. Crowley, G.M. Marion, C.R. de Souza Filho, A.J. Brown, C.M. Weitz, Constraints on the origin and evolution of the layered mound in Gale Crater, Mars using Mars Reconnaissance Orbiter data. Icarus 214(2), 413-432 (2011). doi:10.1016/j.icarus.2011.05.002

A.R. Vasavada, A. Chen, J.R. Barnes, P.D. Burkhart, B.A. Cantor, A.M. Dwyer-Cianciolo, R.L. Fregason, D.P. Hinson, H.L. Justh, D.M. Kass, S.R. Lewis, M.A. Mischna, J.R. Murphy, S.C.R. Rankin, D. Tyler, P.G. Withers, Assessment of environments for Mars Science Laboratory Entry, Descent, and Surface Operations. Space Sci. Rev. (2012). doi:10.1007/s11214-012-9911-3, this issue

D. Vaniman, M.D. Dyar, R. Wiens, A. Ollila, N. Lanza, J. Lasue, J.M. Rhodes, S. Clegg, Ceramic ChemCam calibration targets on Mars Science Laboratory. Space Sci. Rev. (2012). doi:10.1007s11214-012-9886-0

R.C. Wiens, S. Maurice, B. Barraclough, M. Saccoccio (ChemCam team), The ChemCam Instrument Suite on the Mars Science Laboratory (MSL) rover: Body unit and combined systems tests. Space Sci. Rev. (2012). doi:10.1007/s11214-012-9902-4, this issue 